NBER WORKING PAPER SERIES

SUPPLY AND SHORTING IN SPECULATIVE MARKETS

\author{
Marcel Nutz \\ José A. Scheinkman \\ Working Paper 23751 \\ http://www.nber.org/papers/w23751 \\ NATIONAL BUREAU OF ECONOMIC RESEARCH \\ 1050 Massachusetts Avenue \\ Cambridge, MA 02138 \\ August 2017
}

We are indebted to Pete Kyle and Xunyu Zhou for fruitful discussions. Marcel Nutz research was supported by an Alfred P. Sloan Fellowship and NSF Grant DMS-1512900. The views expressed herein are those of the authors and do not necessarily reflect the views of the National Bureau of Economic Research.

NBER working papers are circulated for discussion and comment purposes. They have not been peer-reviewed or been subject to the review by the NBER Board of Directors that accompanies official NBER publications.

(C) 2017 by Marcel Nutz and José A. Scheinkman. All rights reserved. Short sections of text, not to exceed two paragraphs, may be quoted without explicit permission provided that full credit, including $\odot$ notice, is given to the source. 
Supply and Shorting in Speculative Markets

Marcel Nutz and José A. Scheinkman

NBER Working Paper No. 23751

August 2017

JEL No. G12,G4

\begin{abstract}
$\underline{\text { ABSTRACT }}$
We propose a continuous-time model of trading among risk-neutral agents with heterogeneous beliefs. Agents face quadratic costs-of-carry on their positions and as a consequence, their marginal valuation of the asset decreases when the magnitude of their position increases, as it would be the case for risk-averse agents. In the equilibrium models of investors with heterogeneous beliefs that followed the original work by Harrison and Kreps, investors are riskneutral, short-selling is prohibited and agents face a constant marginal cost of carrying positions. The resulting resale option guarantees that the equilibrium price exceeds the price of the asset in a static buy-and-hold model where speculation is ruled out. Our model features three main novelties. First, increasing marginal costs entail that the price depends on the exogenous supply. Second, in addition to the resale option, agents may also value an option to delay, and this may cause the market to equilibrate below the static buy-and-hold price. Third, we introduce the possibility of short-selling; then the resale option for agents with short positions partly compensates the resale option for long agents. We characterize the unique equilibrium of our model through a Hamilton--Jacobi--Bellman equation of a novel form and use it to derive several comparative statics results.
\end{abstract}

Marcel Nutz

Columbia University

1255 Amsterdam Ave

New York, NY 10027

mnutz@stat.columbia.edu

José A. Scheinkman

Department of Economics

Columbia University

New York, NY 10027

and NBER

js3317@columbia.edu 


\title{
Supply and Shorting in Speculative Markets*
}

\author{
Marcel Nutz $\quad$ José A. Scheinkman ${ }^{\ddagger}$
}

August 18, 2017

\begin{abstract}
We propose a continuous-time model of trading among risk-neutral agents with heterogeneous beliefs. Agents face quadratic costs-of-carry on their positions and as a consequence, their marginal valuation of the asset decreases when the magnitude of their position increases, as it would be the case for risk-averse agents. In the equilibrium models of investors with heterogeneous beliefs that followed the original work by Harrison and Kreps, investors are risk-neutral, short-selling is prohibited and agents face a constant marginal cost of carrying positions. The resulting resale option guarantees that the equilibrium price exceeds the price of the asset in a static buy-and-hold model where speculation is ruled out. Our model features three main novelties. First, increasing marginal costs entail that the price depends on the exogenous supply. Second, in addition to the resale option, agents may also value an option to delay, and this may cause the market to equilibrate below the static buy-and-hold price. Third, we introduce the possibility of shortselling; then the resale option for agents with short positions partly compensates the resale option for long agents. We characterize the unique equilibrium of our model through a Hamilton-Jacobi-Bellman equation of a novel form and use it to derive several comparative statics results.
\end{abstract}

\section{Introduction}

As Kindleberger and Aliber (2005) elaborate, many classical economists argued that the purchase of securities for re-sale rather than for investment income was the engine that generated numerous historical asset price bubbles. To explain such speculation in a dynamic equilibrium model, Harrison and Kreps (1978) study risk-neutral agents with fluctuating heterogeneous

\footnotetext{
${ }^{*}$ We are indebted to Pete Kyle and Xunyu Zhou for fruitful discussions.

${ }^{\dagger}$ Columbia University. Research supported by an Alfred P. Sloan Fellowship and NSF Grant DMS-1512900.

${ }^{\ddagger}$ Columbia University, Princeton University and NBER.
} 
beliefs. In their model, long positions can be financed at a constant interest rate and short-selling is ruled out. The buyer of an asset thus acquires both a stream of future dividends and an option to resell which in combination with fluctuating beliefs guarantees that speculators are willing to pay more for an asset than they would pay if they were forced to hold the asset to maturity; that is, risk-neutral investors would pay to be allowed to speculate. Scheinkman and Xiong (2003) added trading costs and showed that these models generate a correlation between trading volume and overpricing, ${ }^{1}$ a characteristic of several of the major bubble episodes in the last three centuries. ${ }^{2}$

Another stylized fact is that bubble implosions often follow increases in supply. For instance, the implosion of the dotcom bubble was preceded by a vast increase in the float of internet shares. ${ }^{3}$ The South Sea bubble lasted less than one year, but in that period the amount of outstanding shares of the South Sea Company more than doubled, and many other joint-stock companies were established. ${ }^{4}$ However, the assumption of risk-neutral investors facing constant marginal costs in the earlier literature on disagreement and bubbles implies that supply is irrelevant for pricing. ${ }^{5}$ Hong et al. (2006) analyzed a two-period model with risk-averse investors where unexpected increases in supply could deflate bubbles. The economics is straightforwardwhen agents are risk-averse, their marginal valuation for a risky asset decreases with the amount they hold.

Short-selling an asset can be seen as a source of additional supply. The collapse of prices for mortgage backed securities in 2007 was preceded by a series of financial innovations that facilitated shorting: the creation of a standardized CDS for MBS in 2005, the introduction of traded indexes for subprime mortgage backed credit derivatives in 2006, and finally the invention of the synthetic CDO that allowed Wall Street to satisfy the global demand for US AAA mortgage bonds without going through the relatively

\footnotetext{
${ }^{1}$ See also Berestycki et al. (2016).

${ }^{2}$ See e.g. Carlos et al. (2006) on the South Sea bubble, Hong and Stein (2007) on the Roaring Twenties, Ofek and Richardson (2003) and Cochrane (2002) on the internet bubble, Xiong and Yu (2011) on the Chinese warrant bubble.

${ }^{3}$ See Ofek and Richardson (2003).

${ }^{4}$ The directors of the SSC understood that the bubble companies competed with the SSC's conversion scheme and could deflate its own bubble. Harris (1994) documents that the Bubble Act of 1720, which banned joint-stock companies except if authorized by Royal Charter, was produced at the behest of the company to limit the competition for capital.

${ }^{5}$ Except for the assumption of positive net supply, issues concerning supply of the asset subject to bubbles are also ignored in the rational bubbles literature (Santos and Woodford (1997)).
} 
slow process of building new housing. ${ }^{6}$ These innovations allowed pessimists to profit from the excessive optimism prevailing among many investors.

We propose a model with a population of investors trading a single asset and aiming to maximize expected cumulative net gains from trade. These investors are risk-neutral and have fluctuating heterogeneous beliefs, including possibly beliefs about future changes in supply. In contrast to the previous literature, shorting is allowed, albeit at a higher cost when compared to going long: holders of short or long positions pay costs which are proportional to the square of their positions. ${ }^{7}$ The two constants of proportionality may be different; the assumptions in the earlier literature correspond to an infinite cost for short positions and zero cost for long positions. The quadratic holding costs reflect monetary costs of holding a long position or borrowing stocks to establish a short position but could also stand in for risks that we do not explicitly model, such as market-wide liquidity shocks that would force agents to liquidate their positions at unfavorable prices. Since costs are quadratic, an agent's marginal valuation of the asset will decrease as their position increases, as it would be the case for risk-averse agents. Thus we view our setup as a tractable alternative to a much more difficult-to-analyze model with risk aversion, with many of the same forces present. In particular, we will show that the supply of the asset does affect prices in our model.

A buyer of the asset today may forecast that at some future date she would be able to sell the asset at a price that would exceed her own valuation of the asset at that date. Because of this resale option, she may be willing to pay more than what she believes is the discounted value of the payoff of an asset. In the classical models, this option causes equilibrium prices to exceed the price that would prevail if re-trading is ruled out - we call the latter the static price for brevity. In addition, there is also an option to delay which has not been highlighted in the earlier literature. A speculator that buys $y$ units today may plan to buy additional units of the asset in future states of the world where there would be a larger difference between the asset price and her marginal valuation for the asset if she holds $y$ units. However, if the cost of holding a long position is zero, this delay option has no impact in equilibrium. The intuition is that, since agents are risk-neutral and there is no cost-of-carry, a buyer of the asset must be indifferent as to the amount of the asset she buys. Hence, the delay option has no value, and in particular,

\footnotetext{
${ }^{6}$ See Scheinkman (2014) for a summary or Lewis (2015) for an excellent detailed account.

${ }^{7}$ For tractability, we assume that costs are a function of the size rather than the value of the position.
} 
the dynamic equilibrium price cannot be smaller than the static price. ${ }^{8}$ In Example 4.6, we show that in the presence of a positive cost, the option to delay may outweigh the resale option and cause the dynamic price to be lower than the static one, even when shorting is prohibited.

When shorting is allowed, the short party also has the corresponding options. An agent that acquires a short position today may forecast that at some future date he would be able to repurchase the asset at a price that would be below his own valuation of the asset at that date. This option to resell a short position, that is the option to cover shorts, in turn, decreases the minimum amount pessimists would be willing to receive for shorting the asset, putting downward pressure on prices. The short party also enjoys an option to delay. In Example 4.9, we show that when the cost of holding a short position is close to the cost of holding a long position, the equilibrium price may be less than the static price. We argue that this happens because the long party values the resale option less than the short party values the repurchase option. This example also illustrates that an unexpected lowering of the cost of shorting can lead to a collapse of an asset price bubble.

\subsection{Overview}

In Section 2 we describe in detail a continuous-time model where $n$ types of agents trade a single asset. The agents disagree on the future evolution of a Markov state whose time $T$ value determines the asset's payoff. In equilibrium, they find a price process for the asset, given by a function of time and Markov state. Types that expect prices to increase on average over the next instant choose to go long, where the amount they go long depends on their expected price changes and the cost of carrying long positions. The other types choose to go short, by amounts that depend on their expected price changes and the cost of carrying short positions. Equilibrium requires that the longs absorb the shorts plus an exogenous supply. Theorem 2.1 shows that there is a unique equilibrium price function and that it can be characterized by a partial differential equation (PDE). This equation is of Hamilton-JacobiBellman-type with a novel form where the optimization runs over the ways to divide the agents into two groups at any time and state; at the optimum, these are the optimists (holding long positions in equilibrium) and the pessimists (holding shorts). The price can also be seen as the value function of a stochastic optimal control problem (Proposition 2.3) where the controller chooses among a set of laws of motion; this is useful for the derivation of

\footnotetext{
${ }^{8}$ This delay option is surely valuable in the "complete markets" model with difference in beliefs analyzed by Dumas et al. (2009), but it is not examined there.
} 
our subsequent results. An economic interpretation of the PDE is given by the solution to a problem of a planner with limited instruments who tries to maximize the initial price (Theorem 2.4). In either problem, a noteworthy feature is that the supply enters mathematically as a running cost (i.e., like intermediate consumption in Merton's problem).

In Section 3 we provide several comparative statics and limiting results. In particular, we show that an increase in supply decreases the equilibrium price and that a decrease (increase) in the cost for long (short) positions increases the price. We also establish that as the cost for long positions converges to zero, the equilibrium price function converges to a function that does not depend on the cost of holding a short position or the amount supplied. Thus, the crucial assumption in the earlier literature is not that shorting is impossible, but that the marginal cost of carrying long positions is constant. On the other hand, as the cost of shorting becomes prohibitive, the equilibrium price converges to a function that does depend on the cost of carrying long positions as well as the exogenous supply of the asset.

In Section 4 we discuss the impact of speculation in our model. To that end, we first characterize the static equilibrium price; that is, the price that prevails when re-trading is not allowed and agents are forced to use buy-andhold strategies. Then we compare this static price with the dynamic one (where re-trading is possible) and in particular we provide examples that illustrate the effect of the resale and delay options, both when shorting is ruled out and when shorting is allowed. The difference between the dynamic price and the static price has been identified as the size of the bubble in the previous literature. With this identification, the examples in this section can be used to illustrate that, in the presence of increasing marginal costsof-carry, lowering the cost for shorts or increasing the cost for long positions can lead not only to a bubble implosion but even to a negative bubble.

The concluding remarks are stated in Section 5 and the proofs for our results in Sections 2-4 are reported in Appendix A.

The assumption that costs are proportional to the square of the position does not accommodate the fact that borrowers of stock typically pay a fee quoted as an annualized percentage of the value of the loaned securities (the rebate rate). This assumption is made to simplify the exposition and allow us to concentrate on the effects of the size of a position on an agent's marginal valuation. In Appendix B we discuss how our results can be generalized if an additional linear term is added to the costs-of-carry. The agents are then divided into three rather than two groups: while strongly optimistic and pessimistic agents hold long and short positions, respectively, an intermediate group expects that prices will not move enough to compensate for 
the first-order costs and stays out. However, the qualitative properties of equilibria remain the same as without the linear term, so we have chosen to use the simpler model in the body of the paper. We also examine in Appendix $\mathrm{C}$ how the model changes if costs of going long and/or short vary across agents. While the structure of the equilibrium remains similar, ceteris paribus, agents with lower costs take larger positions and have a larger influence on the coefficients of the PDE that determines the equilibrium price.

\section{Equilibrium Price}

In this section, we detail our formal setup and show that it leads to a unique equilibrium. The corresponding price is described by a partial differential equation which, in turn, leads us to a stochastic control problem. Finally, an economic interpretation for the latter is provided through a planning problem.

\subsection{Definition of the Equilibrium Price}

We consider $n \geq 1$ types, each with a unit measure of agents, who trade a security over a finite time interval $[0, T]$. For brevity, we will often refer to a type as an agent. The security has a single payoff $f(X(T))$ at the horizon, where $f: \mathbb{R}^{d} \rightarrow \mathbb{R}$ is a bounded continuous function and $X$ is the state process. While there is no ambiguity about $f$, the agents agree to disagree on the evolution of the state process. Mathematically, we take $X$ to be the coordinate-mapping process on the space $\Omega=C\left([0, T], \mathbb{R}^{d}\right)$ of continuous, $d$-dimensional paths, equipped with the canonical filtration and sigma-field. The view of agent $i$ is represented by a probability measure $Q_{i}$ on $\Omega$ under which $X$ follows the stochastic differential equation (SDE)

$$
d X(t)=b_{i}(t, X(t)) d t+\sigma_{i}(t, X(t)) d W_{i}(t), \quad X(0)=x,
$$

where $W_{i}$ is a Brownian motion of dimension $d^{\prime}$ and the functions

$$
b_{i}:[0, T] \times \mathbb{R}^{d} \rightarrow \mathbb{R}^{d}, \quad \sigma_{i}:[0, T] \times \mathbb{R}^{d} \rightarrow \mathbb{R}^{d \times d^{\prime}}
$$

are deterministic. We assume ${ }^{9}$ throughout that (the components of) $b_{i}$ and $\sigma_{i}$ are in $C_{b}^{1,2}$, the set of all bounded continuous functions $g:[0, T] \times$

\footnotetext{
${ }^{9}$ These conditions could be relaxed considerably. The present form allows for a simple exposition avoiding issues of technical nature.
} 
$\mathbb{R}^{d} \rightarrow \mathbb{R}$ whose partial derivatives $\partial_{t} g, \partial_{x_{i}} g, \partial_{x_{i} x_{j}} g$ exist and are continuous and bounded on $[0, T) \times \mathbb{R}^{d}$. Moreover, we assume that ${ }^{10} \sigma_{i}^{2}$ is uniformly parabolic; that is, its eigenvalues are uniformly bounded away from zero. These conditions imply in particular that the SDE (2.1) has a unique (strong) solution.

Notice that we allow for the differences in beliefs to affect both drift and diffusion coefficients. While much of the literature on disagreement in asset markets deals with constant volatility processes and thus naturally assumes that disagreement concerns only drifts, there is plenty of evidence that more complex processes involving stochastic and time-varying volatility are necessary to understand empirical features of asset prices. In this context, it is quite plausible that agents may also differ in their forecasts for the volatility.

Agents trade the security throughout the interval $[0, T]$, at a time $t$ price $P(t)$ to be determined in equilibrium. The agents are subject to an instantaneous cost-of-carry $c$ which is different for long and short positions,

$$
c(y)= \begin{cases}\frac{1}{2 \alpha_{+}} y^{2}, & y \geq 0, \\ \frac{1}{2 \alpha_{-}} y^{2}, & y<0 .\end{cases}
$$

Here the (inverse) cost coefficients $\alpha_{ \pm}$are given constants satisfying

$$
0<\alpha_{-} \leq \alpha_{+},
$$

meaning that the cost of shorting is higher than the cost of going long. An admissible portfolio $\Phi$ for an agent is a bounded ${ }^{11}$, progressively measurable process, and we write $\mathcal{A}$ for the collection of all these portfolios. The value $\Phi(t)$ indicates the number of units of the security held by the agent at time $t$, and this number can be negative in the case of a short position. Given a (semimartingale) price process $P$, agent $i$ seeks to maximize the expected net payoff ${ }^{12}$

$$
E_{i}\left[\int_{0}^{T} \Phi(t) d P(t)-\int_{0}^{T} c(\Phi(t)) d t\right]
$$

here the first integral represents the profit-and-loss from trading and the second integral is the cumulative cost-of-carry incurred. Criterion (2.3) can

\footnotetext{
${ }^{10}$ Given a matrix $A$, we write $A^{2}$ for the product $A A^{\top}$ of $A$ with its transpose $A^{\top}$.

${ }^{11}$ Boundedness could be replaced by suitable integrability conditions without altering our results.

${ }^{12}$ To ensure that the expectation is well-defined a priori, we set $E_{i}[Y]:=-\infty$ whenever $E_{i}[\min \{0, Y\}]=-\infty$, for any random variable $Y$. For the processes $P$ that occur in our results below, (2.3) will be finite for any $\Phi \in \mathcal{A}$.
} 
be rationalized by assuming that agents have access to borrowing and lending at a zero interest rate and that the cost function $c$ is measured in the unit of account but it can also be taken as a primitive utility function. ${ }^{13}$ An admissible portfolio $\Phi_{i}$ will be called optimal for agents of type $i$ if it maximizes (2.3) over all $\Phi \in \mathcal{A}$. We will examine symmetric equilibria in which agents of the same type choose the same portfolio.

As the final input of our model, we introduce a nonnegative, progressively measurable process $S$ representing exogenous supply. The latter is owned by third parties that supply their endowment inelastically. ${ }^{14}$ We assume that $S(t)=s(t, X(t))$ for a deterministic and nonnegative supply function $s \in C_{b}^{1,2}{ }^{15}$ Let $P$ be a continuous, progressively measurable process which is a semimartingale with $P(T)=f(X(T))$ a.s. under all $Q_{i}$. We say that $P$ is an equilibrium price if there exist admissible portfolios $\Phi_{i}, i \in\{1, \ldots, n\}$ such that $\Phi_{i}$ is optimal for agent $i$ and the market clearing condition

$$
\sum_{i=1}^{n} \Phi_{i}(t)=S(t)
$$

holds. We are interested in Markovian equilibria; that is, equilibrium prices of the form $P(t)=v(t, X(t))$ for a deterministic function $v$ which we refer to as an equilibrium price function.

\subsection{Existence and PDE for the Equilibrium Price}

The following notation will be useful to state our first result. Given $v \in C_{b}^{1,2}$, we define the function $\mathcal{L}^{i} v$ by

$$
\mathcal{L}^{i} v(t, x)=\partial_{t} v(t, x)+b_{i} \partial_{x} v(t, x)+\frac{1}{2} \operatorname{Tr} \sigma_{i}^{2} \partial_{x x} v(t, x) .
$$

Here $\partial_{x} v$ denotes the gradient vector, $\partial_{x x} v$ the Hessian matrix, and $\operatorname{Tr} \sigma_{i}^{2} \partial_{x x} v$ is the trace of the matrix $\sigma_{i}^{2} \partial_{x x} v$; that is, the sum of the entries on the diagonal. Finally, for a subset $I \subseteq\{1, \ldots, n\}$, we denote by $I^{c}=\{1, \ldots, n\} \backslash I$ the complement and by $|I|$ the number of elements in $I$.

Before stating the characterization of equilibria it is worthwhile to examine the homogeneous case where all agents have the same views, represented by coefficients $b_{i}=b$ and $\sigma_{i}=\sigma$. Thus, $\mathcal{L}^{i} v(t, x)=\mathcal{L} v(t, x)$

\footnotetext{
${ }^{13}$ By adding notation we could accommodate a constant non-zero interest rate or discount rate.

${ }^{14}$ Since the utility function in $(2.3)$ is separable, the equilibrium price is invariant to endowments. Hence, we could have alternatively assumed an arbitrary ownership structure for the endowment across the types of investors - we opted for the simpler presentation.

${ }^{15}$ Notice that this formalism allows for the payoff $f(X(T))$ to depend on $S(T)$.
} 
is also independent of $i$. The quadratic costs imply that the optimal positions are unique and identical across agents; in particular, there is no short-selling in equilibrium. Positions are determined by the first-order condition $\Phi_{i}(t)=\alpha_{+} \mathcal{L} v(t, X(t))$ and thus market clearing requires that $\alpha_{+} \mathcal{L} v(t, X(t))=S(t) / n$ or

$$
\partial_{t} v(t, x)+b(t, x) \partial_{x} v(t, x)+\frac{1}{2} \operatorname{Tr} \sigma^{2}(t, x) \partial_{x x} v(t, x)-\frac{s(t, x)}{n \alpha_{+}}=0 .
$$

Notice that supply enters as a running cost: the equilibrium price must compensate for the cost-of-carry.

The following theorem characterizes equilibria in the general case of heterogeneous agents. In contrast to the above, the PDE becomes nonlinear and depends on the cost of shorting.

Theorem 2.1. (i) There exists a unique equilibrium price function $v \in$ $C_{b}^{1,2}$. The corresponding optimal portfolios are unique ${ }^{16}$ and given by $\Phi_{i}(t)=$ $\phi_{i}(t, X(t))$, where

$$
\phi_{i}(t, x)=\alpha_{\operatorname{sign}\left(\mathcal{L}^{i} v(t, x)\right)} \mathcal{L}^{i} v(t, x) .
$$

(ii) The function $v \in C_{b}^{1,2}$ can be characterized as the unique solution of the PDE

$\partial_{t} v(t, x)+\sup _{I \subseteq\{1, \ldots, n\}}\left(\mu_{I}(t, x) \partial_{x} v(t, x)+\frac{1}{2} \operatorname{Tr} \Sigma_{I}^{2}(t, x) \partial_{x x} v(t, x)-\kappa_{I}(t, x)\right)=0$

on $[0, T) \times \mathbb{R}^{d}$ with terminal condition $v(T, x)=f(x)$, where the supremum is taken over all subsets $I \subseteq\{1, \ldots, n\}$ and the coefficients are defined as

$$
\begin{gathered}
\mu_{I}(t, x)=\frac{\alpha_{-}}{|I| \alpha_{-}+\left|I^{c}\right| \alpha_{+}} \sum_{i \in I} b_{i}(t, x)+\frac{\alpha_{+}}{|I| \alpha_{-}+\left|I^{c}\right| \alpha_{+}} \sum_{i \in I^{c}} b_{i}(t, x), \\
\Sigma_{I}^{2}(t, x)=\frac{\alpha_{-}}{|I| \alpha_{-}+\left|I^{c}\right| \alpha_{+}} \sum_{i \in I} \sigma_{i}^{2}(t, x)+\frac{\alpha_{+}}{|I| \alpha_{-}+\left|I^{c}\right| \alpha_{+}} \sum_{i \in I^{c}} \sigma_{i}^{2}(t, x), \\
\kappa_{I}(t, x)=\frac{s(t, x)}{|I| \alpha_{-}+\left|I^{c}\right| \alpha_{+}} .
\end{gathered}
$$

For the sake of brevity, we will often suppress the arguments $(t, x)$ in our notation for the statements below. To explain the formulas (2.6) and (2.7)

\footnotetext{
${ }^{16}$ Uniqueness is understood up to $\left(Q_{i} \times d t\right)$-nullsets.
} 
for $\mu_{I}$ and $\Sigma_{I}$, let $I \subseteq\{1, \ldots, n\}$; we think of $I$ and $I^{c}$ as two groups of agents. We may first form the average volatilities within each set,

$$
\sigma_{I}^{2}=\frac{1}{|I|} \sum_{i \in I} \sigma_{i}^{2}, \quad \bar{\sigma}_{I^{c}}^{2}=\frac{1}{\left|I^{c}\right|} \sum_{i \in I^{c}} \sigma_{i}^{2}
$$

and then take a weighted average between these two to obtain

$$
\Sigma_{I}^{2}=\frac{|I| \alpha_{-}}{|I| \alpha_{-}+\left|I^{c}\right| \alpha_{+}} \sigma_{I}^{2}+\frac{\left|I^{c}\right| \alpha_{+}}{|I| \alpha_{-}+\left|I^{c}\right| \alpha_{+}} \sigma_{I^{c}}^{2},
$$

where the weights contain the cost coefficients $\alpha_{ \pm}$that each type is subject to - their interpretation will be discussed in more detail in Theorem 2.4 below. The coefficients $\mu_{I}$ are formed from the initial drifts $b_{i}$ in the same fashion. The running cost $\kappa_{I}$ of (2.8) depends linearly on the exogenous supply $s$ which is divided by a weighted sum of the cost coefficients, the weights being the size of set $I$ and its complement $I^{c}$, respectively. Since $\alpha_{-} \leq \alpha_{+}$, the cost increases with the number $|I|$ of types in the group $I$.

Remark 2.2. The equilibrium price $v(t, x)$ is 0-homogeneous in $\left(\alpha_{-}, \alpha_{+}, s\right)$, indicating that supply and costs are closely linked in our model. That is, if these parameters are replaced by $\left(\lambda \alpha_{-}, \lambda \alpha_{+}, \lambda s\right)$ for some $\lambda>0$, the price does not change. This follows from Theorem 2.1 (ii) after observing that the coefficients $\mu_{I}, \Sigma_{I}$ and $\kappa_{I}$ are invariant under this substitution. For instance, increasing the supply by a factor $\lambda$ has the same effect on the price as increasing both costs-of-carry by the same factor: $\left(\alpha_{-}, \alpha_{+}, \lambda s\right)$ and $\left(\lambda^{-1} \alpha_{-}, \lambda^{-1} \alpha_{+}, s\right)$ produce the same equilibrium price.

In the special case $s=0$, the homogeneity entails that the price depends on $\left(\alpha_{-}, \alpha_{+}\right)$only through the ratio $\alpha_{+} / \alpha_{-}$; in fact, we will show in Proposition 3.2 below that the price is increasing with respect to $\alpha_{+} / \alpha_{-}$.

\subsection{Optimal Control Representation}

The PDE (2.5) is the Hamilton-Jacobi-Bellman equation of a stochastic optimal control problem where the controller can choose a subset $I \subseteq\{1, \ldots, n\}$ at any time and state, and that choice determines the instantaneous drift and volatility coefficients $\mu_{I}$ and $\Sigma_{I}$ as well as the running cost $\kappa_{I}$.

To formulate this problem precisely, consider a filtered probability space carrying a $d^{\prime}$-dimensional Brownian motion $W$ and let $\Theta$ be the collection of all progressively measurable processes $\mathcal{I}$ with values in the family of all subsets of $\{1, \ldots, n\} .{ }^{17}$ For each $\mathcal{I} \in \Theta$, let $X_{\mathcal{I}}^{t, x}(r), r \in[t, T]$ be the solution

\footnotetext{
${ }^{17}$ While this collection of control processes appears somewhat non-standard, there is no difficulty involved in defining it - this family of subsets is simply a discrete set with $2^{n}$ elements; it can be identified with $\{0,1\}^{n}$.
} 
of the controlled SDE

$$
d X(r)=\mu_{\mathcal{I}(r)}(r, X(r)) d r+\Sigma_{\mathcal{I}(r)}(r, X(r)) d W(r), \quad X(t)=x
$$

on the time interval $[t, T]$. It follows from the assumptions on the coefficients $b_{i}, \sigma_{i}$ that this SDE with random coefficients has a unique strong solution. Therefore, we may consider the control problem

$$
V(t, x)=\sup _{\mathcal{I} \in \Theta} E\left[f\left(X_{\mathcal{I}}^{t, x}(T)\right)-\int_{0}^{T} \kappa_{\mathcal{I}(r)}\left(r, X_{\mathcal{I}}^{t, x}(r)\right) d r\right]
$$

for $(t, x) \in[0, T] \times \mathbb{R}^{d}$, which gives rise to a second characterization for the equilibrium price function $v$.

Proposition 2.3. The equilibrium price function $v$ from Theorem 2.1 coincides with the value function $V$ of (2.10). Moreover, an optimal control for $(2.10)$ is given by $\mathcal{I}_{*}(t)=I_{*}(t, X(t))$, where

$$
I_{*}(t, x)=\left\{i \in\{1, \ldots, n\}: \mathcal{L}^{i} v(t, x)<0\right\} .
$$

Proposition 2.3 shows the equivalence between the equilibrium price $v$ and the solution to an abstract control problem where the controller chooses at each instant one among $2^{n}$ possible laws of motion and associated cost functions. This will be mathematically useful later on but has no direct economic interpretation. Next, we introduce a planner with limited instruments, or a principal-agent problem, to provide this interpretation.

\subsection{A Planner with Limited Instruments}

In this section, we characterize the equilibrium price function as the optimal value for a planner with limited instruments. We take the stochastic control representation (2.10) as our starting point and first remark that the value of $V(t, x)$ does not change if we replace $\Theta$ by the set of all feedback controls; that is, controls of the form $\mathcal{I}(r)=I(r, X(r))$ for some deterministic (and measurable) function $I$. This follows, for instance, from the fact that the optimal control in Proposition 2.3 is of this form.

Consider a planner who can choose to tax or subsidize specific types at each date and event. More specifically the planner assigns to each type at each date event pair, a "total cost coefficient" $\alpha_{i} \in\left\{\alpha_{-}, \alpha_{+}\right\}$. If an agent of a type that is assigned $\alpha_{+}$decides to go short $y$ units, she will be subsidized so that her effective cost is $\frac{1}{2 \alpha_{+}} y^{2}$. If an agent of a type that is assigned $\alpha_{-}$ decides to go long $y$ units, she will be taxed so that her effective cost is $\frac{1}{2 \alpha_{-}} y^{2}$. 
That is, types are taxed or subsidized so that their cost is not affected by the sign of their chosen position in the asset. Otherwise every agent is free to choose a position at each date event pair taking prices as given and the market settles on prices that equilibrate supply and demand for the asset. Aggregate taxes collected by the planner are returned lump-sum to agents. We postulate that the planner's objective is to maximize the initial price, perhaps because she wants to maximize the welfare of the time-zero sellers who inelastically supply their holdings.

Since taxes are returned lump-sum, given the costs and tax-subsidy scheme faced by agents at each $(t, x)$ their objective is to maximize the expected (net of lump-sum transfers) payoff

$$
E_{i}\left[\int_{0}^{T} \Phi(t) d P(t)-\int_{0}^{T} c_{i}(t, X(t), \Phi(t)) d t\right]
$$

which is similar to (2.3), except that now the cost-of-carry is given by the assigned coefficient,

$$
c_{i}(t, x, y)=\alpha_{i}(t, x) y^{2},
$$

irrespectively of the position being long or short. We may then again look for an equilibrium price $P(t)=v(t, X(t))$ with $P(T)=f(X(T))$ a.s. under all $Q_{i}$ and corresponding optimal portfolios $\Phi_{i}$ that clear the market. Of course, all these quantities depend on the assignment made by the planner, which we summarize as a mapping

$$
I(t, x)=\left\{i \in\{1, \ldots, n\}: \alpha_{i}(t, x)=\alpha_{-}\right\} .
$$

That is, $I$ designates the group of agents with effective cost coefficient $\alpha_{-}$, and correspondingly agents in $I^{c}$ have $\alpha_{+}$. The following result establishes the connection with the control problem (2.10) if the assignment $I$ is seen as a control $\mathcal{I}(t)=I(t, X(t))$ in feedback form.

Theorem 2.4. (i) For any assignment $\mathcal{I}(t)=I(t, X(t))$ of the planner, there exists a unique equilibrium price process $P_{\mathcal{I}}(t)=v_{\mathcal{I}}(t, X(t))$ with $v_{\mathcal{I}} \in$ $C_{b}^{1,2}$, and $v_{\mathcal{I}}$ is given by

$$
v_{\mathcal{I}}(t, x)=E\left[f\left(X_{\mathcal{I}}^{t, x}(T)\right)-\int_{0}^{T} \kappa_{\mathcal{I}(r)}\left(r, X_{\mathcal{I}}^{t, x}(r)\right) d r\right] .
$$

(ii) If the planner's aim is to maximize the price $P_{\mathcal{I}}(0)=v_{\mathcal{I}}(0, x)$, then the assignment $(2.11)$ is optimal and $V(0, x)$ of $(2.10)$ is the optimal price. ${ }^{18}$

\footnotetext{
${ }^{18}$ The analogous result holds for maximizing the price at a positive time $t \in(0, T)$ instead of $t=0$.
} 
Thus, in the optimal assignment, no taxes or subsidies are collected, and in the resulting equilibrium, agents assigned $\alpha_{-}$go short and agents assigned $\alpha_{+}$go long. In particular, the planner penalizes pessimists by assigning the higher cost-of-carry to short positions, which then leads to the maximal price that the planner can produce given her constraints.

\section{Comparative Statics and Limiting Cases}

In the first part of this section, we establish comparative statics with respect to the supply and cost parameters, showing that our model has the desired monotonicity properties. In the second part, we analyze the limit $\alpha_{+} \rightarrow \infty$ where there is no cost-of-carry for long positions, as well as the limit $\alpha_{-} \rightarrow 0$ where short positions are ruled out.

\subsection{Comparative Statics}

We start with the dependence on the supply.

Proposition 3.1. The equilibrium price function $v$ is monotone decreasing with respect to the supply function s: the price decreases with an increase in supply.

Next, we turn to the cost parameters $\alpha_{-}$and $\alpha_{+}$. The following shows that the equilibrium price is decreasing with respect to the cost-of-carry for long positions and increasing with respect to the cost for short positions.

Proposition 3.2. The equilibrium price function $v$ is

(i) increasing with respect to $\alpha_{+}$,

(ii) decreasing with respect to $\alpha_{-}$,

(iii) increasing with respect to the quotient $\alpha_{+} / \alpha_{-}$if $s \equiv 0$.

The following is a partial extension of (iii) to the case of non-zero supply which is useful if $\alpha_{-}$and $\alpha_{+}$are varied simultaneously.

Remark 3.3. Let $\alpha_{-} \leq \alpha_{+}$and $\alpha_{-}^{\prime} \leq \alpha_{+}^{\prime}$ be two pairs of cost coefficients and let $v$ and $v^{\prime}$ be the corresponding equilibrium price functions. If the coefficients satisfy $\alpha_{+} / \alpha_{-} \leq \alpha_{+}^{\prime} / \alpha_{-}^{\prime}$ and $\alpha_{-} \leq \alpha_{-}^{\prime}$, then $v \leq v^{\prime}$.

For instance, it follows that if the costs-of-carry for long and short positions are increased by a common factor, then the price decreases. 


\subsection{Limiting Models}

We discuss two limits for the cost coefficients which help to understand the relationship between our model and the earlier ones mentioned in the Introduction. To make the dependence on the parameters explicit, we denote by $v^{\alpha_{-}, \alpha_{+}}(t, x)$ the equilibrium price function $v(t, x)$ for $\alpha_{-}, \alpha_{+}$as introduced in Theorem 2.1.

\subsubsection{Zero Cost for Long Positions}

We first consider the limit $\alpha_{+} \rightarrow \infty$ when the cost-of-carry for long positions tends to zero.

Proposition 3.4. As $\alpha_{+} \rightarrow \infty$, the function $v^{\alpha_{-}, \alpha_{+}}$converges to the unique solution $v^{\infty} \in C_{b}^{1,2}$ of the PDE

$$
\partial_{t} v+\sup _{i \in\{1, \ldots, n\}}\left(b_{i} \partial_{x} v+\frac{1}{2} \operatorname{Tr} \sigma_{i}^{2} \partial_{x x} v\right)=0
$$

with terminal condition $v(T, x)=f(x)$; in particular, $v^{\infty}$ is independent of $\alpha_{-}$and $s$. The convergence is locally uniform in $(t, x)$, and monotone increasing as $\alpha_{+} \uparrow \infty$.

We now discuss the limiting model that arises in Proposition 3.4; that is, a variant of our model with no cost-of-carry for long positions. We state these results without proofs since the mathematical arguments are very similar to the proof of Theorem 2.1.

The limiting model has an equilibrium price function $v:=v^{\infty}$ that is unique and independent of the supply $s$ and the cost coefficient $\alpha_{-}$for short positions. The optimal portfolios $\Phi_{i}(t)=\phi_{i}(t, X(t))$ for the equilibrium, however, do depend on $s$ and $\alpha_{-}$. Given $(t, x)$, we may distinguish two cases for an agent $i$. If the index $i$ is not a maximizer in (3.1), or equivalently if $\mathcal{L}^{i} v(t, x)<0$, then

$$
\phi_{i}(t, x)=\alpha_{-} \mathcal{L}^{i} v(t, x)
$$

as in (2.4); in particular, agent $i$ holds a short position. On the other hand, the "most optimistic" agents $i$ which attain the maximum, or equivalently satisfy $\mathcal{L}^{i} v(t, x)=0$, only require that $\phi_{i}(t, x)$ be nonnegative - they are invariant with respect to the amount as long as the position is nonnegative. In equilibrium, the amounts held by the most optimistic types are set by the market clearing condition: if there is only one maximizer $i$, then

$$
\phi_{i}(t, x)=s(t, x)-\sum_{j \in\{1, \ldots, n\} \backslash\{i\}} \alpha_{-} \mathcal{L}^{j} v(t, x)
$$


is uniquely determined - the most optimistic type absorbs all the exogenous supply plus the short positions of the other agents. This explains why the price does not depend on $s$ and $\alpha_{-}$; in fact, the price is solely determined by the characteristics of the most optimistic type. If there is more than one maximizer $i$, meaning that several types are equally optimistic, then the optimal portfolios are not unique - any distribution of the available amount (supply plus short positions) over those types gives an optimal allocation.

The properties described above for $\alpha_{+}=\infty$ continue to hold if in addition $\alpha_{-}=0$; i.e., when there is no cost for long positions and short positions are prohibited. In particular, all but the most optimistic agents hold a flat position, and only the most optimistic characteristics play a role in determining the price. ${ }^{19}$ Thus, we retrieve the results of previous models with risk-neutral agents in this limiting regime, as mentioned in the Introduction.

Remark 3.5. Proposition 3.4 shows that in the limiting case $\alpha_{+}=\infty$, the price is determined by the most optimistic agent at any state. This may be contrasted with the opposite extreme case where the cost coefficients $\alpha_{+}=\alpha_{-}$are equal. Then, the drift and volatility coefficients

$$
\mu:=\mu_{I}=\frac{1}{n} \sum_{i=1}^{n} b_{i}, \quad \Sigma^{2}:=\Sigma_{I}^{2}=\frac{1}{n} \sum_{i=1}^{n} \sigma_{i}^{2}
$$

are independent of $I$ and equal to the arithmetic average of the coefficients in the agents' models, meaning that all agents contribute equally to the price. The running cost is $\kappa:=\kappa_{I}=s /\left(n \alpha_{+}\right)$. Thus, the PDE (2.5) becomes linear,

$$
\partial_{t} v+\frac{1}{n} \sum_{i=1}^{n} b_{i} \partial_{x} v(t, x)+\frac{1}{2 n} \sum_{i=1}^{n} \operatorname{Tr} \sigma_{i}^{2} \partial_{x x} v-\frac{s}{n \alpha_{+}}=0
$$

and the equilibrium price is

$$
v(t, x)=E\left[f\left(X^{t, x}(T)\right)-\int_{0}^{T} s\left(r, X^{t, x}(r)\right) /\left(n \alpha_{+}\right) d r\right]
$$

where $X^{t, x}$ follows the $\operatorname{SDE}(2.9)$ with coefficients $\mu$ and $\Sigma$. That is, the equilibrium price is simply the expected value of the security under the averaged coefficients of the agents, minus a cost term related to the supply.

\footnotetext{
${ }^{19}$ This model was studied in detail by Muhle-Karbe and Nutz (2016), under the the assumption of a unit net supply.
} 


\subsubsection{Infinite Cost for Short Positions}

We now discuss the limit $\alpha_{-} \rightarrow 0$; that is, the cost-of-carry for short positions tends to infinity.

Proposition 3.6. As $\alpha_{-} \rightarrow 0$, the function $v^{\alpha_{-}, \alpha_{+}}$converges to the unique solution $v^{0, \alpha_{+}} \in C_{b}^{1,2}$ of the PDE

$$
\partial_{t} v+\sup _{\emptyset \neq J \subseteq\{1, \ldots, n\}}\left(\frac{1}{|J|} \sum_{i \in J} b_{i} \partial_{x} v+\frac{1}{2} \operatorname{Tr} \frac{1}{|J|} \sum_{i \in J} \sigma_{i}^{2} \partial_{x x} v-\frac{s}{|J| \alpha_{+}}\right)=0
$$

with terminal condition $v(T, x)=f(x)$. In the special case where $s=0$, this $P D E$ coincides with (3.1) and in particular the solution $v^{0, \alpha_{+}}=v^{\infty}$ is independent of $\alpha_{+}$. The convergence is locally uniform in $(t, x)$, and monotone increasing if $\alpha_{-} \downarrow 0$.

The limiting model that arises in Proposition 3.6, where there is an infinite cost for short positions, corresponds to a prohibition of shorting-selling. This model has a unique equilibrium price function $v:=v^{0, \alpha_{+}}$, and in contrast to the above, the price does depend on the supply $s$ and the cost coefficient $\alpha_{+}$for long positions. The optimal portfolios $\Phi_{i}(t)=\phi_{i}(t, X(t))$ are unique and given by

$$
\phi_{i}(t, x)= \begin{cases}\alpha_{+} \mathcal{L}^{i} v(t, x), & \mathcal{L}^{i} v(t, x) \geq 0 \\ 0, & \mathcal{L}^{i} v(t, x)<0\end{cases}
$$

and both cases may indeed occur. At every state $(t, x)$, we can think of the types as being divided into a group $J=\left\{i \in\{1, \ldots, n\}: \mathcal{L}^{i} v(t, x) \geq 0\right\}$ of relatively optimistic agents and the complement $J^{c}$ of pessimists. We have $J \neq \emptyset$ by market clearing. While the agents in $J$ hold positions of different magnitude depending on how optimistic they are, the entire group $J$ determines the price. Agents in $J^{c}$, however, hold zero units and their precise characteristics do not enter the formation of the price. For instance, if we replace a pessimistic type $i \in J^{c}$ by an even more pessimistic type, the equilibrium price will not change.

We remark that the case $s=0$ is degenerate in the limiting model: while the above assertions are valid, the portfolios are in fact given by $\Phi_{i} \equiv 0$ for all $i$. Indeed, the PDE implies $\mathcal{L}^{i} v(t, x) \leq 0$. Alternately, this follows from the fact that there is neither supply nor shorting in this case. 


\section{Speculation}

In this section, we highlight the impact of non-linear costs-of-carry and shortselling on the pricing mechanism by comparing the above "dynamic" equilibrium price at time $t=0$ with a "static" equilibrium price; that is, an equilibrium without speculation. We shall see that, as in previous models, the dynamic price dominates the static price when cost-of-carry and shortselling are removed from our model. This can be attributed to the resale option. However, we show that the cost-of-carry (i.e., risk aversion) gives rise to a delay effect that may act in opposition to the resale option and reverse the order of the prices in extreme cases - even if short-selling is prohibited. Moreover, we illustrate that the possibility of short-selling tends to depress the dynamic price, as it gives rise to a repurchase option for pessimists.

\subsection{Equilibrium without Speculation}

Let us consider a situation where trading occurs only at the initial time $t=0$; that is, agents are forced to use buy-and-hold strategies and speculation is ruled out. The agents use the same models $Q_{i}$ for the dynamics $(2.1)$ of the state process $X$ and maximize the same expected net payoff (2.3). However, the admissible portfolios $\Phi$ are restricted to be constant, and we will use the letter $q$ to denote a generic portfolio. This market can only clear if the exogenous supply $S \equiv s$ is constant, so we restrict our attention to that case. A static equilibrium price is defined like the dynamic equilibrium price above, except that we only look for a constant $p_{\text {sta }} \in \mathbb{R}$ at time $t=0$ at which the trading happens.

Proposition 4.1. (i) There exists a unique static equilibrium price and it is given by

$$
p_{\text {sta }}=\max _{I \subseteq\{1, \ldots, n\}}\left(\frac{\alpha_{-}}{|I| \alpha_{-}+\left|I^{c}\right| \alpha_{+}} \sum_{i \in I} e_{i}+\frac{\alpha_{+}}{|I| \alpha_{-}+\left|I^{c}\right| \alpha_{+}} \sum_{i \in I^{c}} e_{i}-\frac{s T}{|I| \alpha_{-}+\left|I^{c}\right| \alpha_{+}}\right),
$$

where $e_{i}=E_{i}[f(X(T))]$. The corresponding optimal static portfolios are unique and given by

$$
q_{i}=\alpha_{\text {sign }\left(e_{i}-p_{\text {sta }}\right)} T^{-1}\left(e_{i}-p_{\text {sta }}\right) .
$$

We may observe that the formula (4.1) for the static price is reminiscent of the PDE (2.5) for the dynamic price; however, the averaging of the models of the different types now occurs at the level of the expected values $e_{i}=$ 
$E_{i}[f(X(T))]$ instead of the drift and volatility coefficients $b_{i}, \sigma_{i}$. Informally, we may think of the PDE as describing a repeated version of the static problem over infinitesimal intervals.

In parallel to our analysis above, we can consider limiting cases for the cost coefficients in the static case. The limiting models have interpretations analogous to the dynamic limiting models in Section 3.2, so we confine ourselves to stating the formulas for the limiting prices. We denote by $p_{\text {sta }}^{\alpha_{-}, \alpha_{+}}$ the static equilibrium price for cost parameters $\alpha_{-}, \alpha_{+}$and initial value $X(0)=x$ as given by (4.1).

Proposition 4.2. (i) In the limit $\alpha_{+} \rightarrow \infty$ with zero cost for long positions, the price $p_{\mathrm{sta}}^{\alpha_{-}, \alpha_{+}}$converges to

$$
p_{\mathrm{sta}}^{\infty}=\max _{i \in\{1, \ldots, n\}} E_{i}[f(X(T))] .
$$

(ii) In the limit $\alpha_{-} \rightarrow 0$ with infinite cost for short positions, the price $p_{\mathrm{sta}}^{\alpha_{-}, \alpha_{+}}$ converges to

$$
p_{\mathrm{sta}}^{0, \alpha_{+}}=\max _{\emptyset \neq J \subseteq\{1, \ldots, n\}}\left(\frac{1}{|J|} \sum_{i \in J} E_{i}[f(X(T))]-\frac{s T}{|J| \alpha_{+}}\right) .
$$

\subsection{Resale and Delay Options}

Next, we compare the dynamic equilibrium price $p_{\text {dyn }}:=P(0)$ at time $t=0$ with the static equilibrium price $p_{\text {sta }}$. For the latter to be well-defined, we assume throughout that the supply $s$ is constant. We discuss two options that are present under dynamic trading and are valued by agents - the resale and delay options - and the effect on prices of eliminating these options by forcing agents to trade only at time zero. In particular, we shall see that the ordering of $p_{\text {dyn }}$ and $p_{\text {sta }}$ may be different than in the earlier models.

Previous papers, starting with Harrison and Kreps (1978), considered models with risk-neutral agents that face a constant marginal cost-of-carry for long positions (the interest rate) and cannot sell short. In such models, it is known that the dynamic equilibrium price exceeds the static one, and the difference is attributed to the "resale option." Indeed, the possibility of reselling the asset increases the price - agents may want to buy today in order to resell to agents that are more optimistic tomorrow. In these "classical" models, agents may also choose to delay their purchases, because they believe that while today's price exceeds their marginal valuation, in some states of the world tomorrow's prices may be more favorable. This possibility however does not alter the ranking between the dynamic and static equilibrium prices. 
Indeed, since agents are risk-neutral and the marginal cost of carrying a long position is independent of the size of the position, we may assume generically that only one type $i$ would acquire the asset in the static equilibrium. When re-trading is allowed, $i$ 's marginal valuation for holding the full supply of the asset at time zero is at least as large, since an agent can always choose a buy-and-hold strategy. As the market price must exceed the marginal valuation of any type, the dynamic equilibrium price must exceed the static equilibrium price.

The next two results confirm this intuition by showing how this mechanism carries over to limiting cases of our model. First, we show that when there is no cost for long positions, the dynamic price exceeds the static one. This holds even when shorting is allowed, because in this extreme case, only the most optimistic agents contribute to the price formation, just like in the classical models (see also Propositions 3.4 and 4.2).

Proposition 4.3. In the limit $\alpha_{+} \rightarrow \infty$, the dynamic equilibrium price dominates the static price: $p_{\mathrm{dyn}}^{\infty} \geq p_{\mathrm{sta}}^{\infty}$.

Next, we show that if short-sales are prohibited and if in the static equilibrium only one type holds the asset, the dynamic equilibrium price again exceeds the static price, even when longs pay a cost-of-carry.

Proposition 4.4. In the limit $\alpha_{-} \rightarrow 0$ with no short-selling, suppose that a single type $i$ holds the entire market in the static equilibrium; that is, $q_{i}=s$ and $q_{j}=0$ for $j \neq i$. Then, the dynamic equilibrium price dominates the static price: $p_{\text {dyn }}^{0, \alpha_{+}} \geq p_{\text {sta }}^{0, \alpha_{+}}$.

We now turn to our model with positive costs-of-carry. Here, the same options to resell and to delay are present, but the effects are more subtle. The option to delay now has an effect on equilibrium prices because the marginal valuation of buyers varies with the size of their position. More importantly, trading may occur in the dynamic equilibrium even though one type remains the most optimistic. Indeed, in the classical models (and the limiting model of Proposition 3.4) the most optimistic type always holds the full supply and trading requires that relative optimism changes sign. When there is a costof-carry for long positions, the magnitude of relative optimism determines equilibrium holdings - it is no longer true that a less optimistic type would always hold a non-positive amount. Example 4.6 below illustrates that the delay option may have an important impact on prices and even reverse the ordering of dynamic and static prices.

If shorting is allowed, buying today in order to resell needs to be compared with entering a short position tomorrow. The choice will depend, 
among other factors, on the costs-of-carry for long and short positions. The option to resell a short position - that is, the option to cover shorts - in turn, decreases the minimum amount pessimists would be willing to receive for shorting the asset, putting downward pressure on prices. Shorts also may exercise the option to delay by building up a short position over time. Example 4.9 below illustrates how the ordering of dynamic and static prices can be reversed if the cost of shorting is sufficiently low.

Remark 4.5. In the remainder of this section, we use a quadratic payoff function $f$ as this is convenient to obtain explicit formulas. Of course, that violates our standing assumption that $f$ is bounded. However, our results still apply with the appropriate modifications; in particular, the equilibrium price function $v$ and the admissible portfolios $\phi_{i}$ are of polynomial growth instead of being bounded. The formulas in our examples can also be verified by direct calculation.

\subsection{Illustrating the Effect of the Delay Option}

In this section, we show that the static price may dominate the dynamic one even when short-selling is prohibited. That cannot be explained with a resale option; instead, it highlights the delay option. Consider first the dynamic equilibrium and suppose that type $i$ expects with high probability that their portfolio $\Phi_{i}(t)$ will increase over time. In the static equilibrium, we eliminate the possibility of changing the portfolio in the future. Thus, an agent of type $i$ will consider anticipating the increase of the portfolio by augmenting the position already at time $t=0$, and if the additional expected gains outweigh the additional costs-of-carry, the agent will indeed have a higher demand at the previous price $p_{\text {dyn }}$. Then, the equilibrium can adjust in two ways. First, other types may reduce their positions at the same price. For instance, this may happen because they are anticipating a decrease in position or because they are indifferent to the amount they are holding (see also Example 4.8 below). Second, if all types have the same or even a higher demand ${ }^{20}$ in the static case, the equilibrium must adjust by an increase in price, and then the static equilibrium price may exceed the dynamic one, despite the resale option. In practice, we may often observe a mixture of these two cases: other types may reduce their positions after an increase in price.

\footnotetext{
${ }^{20}$ Note that since the types disagree, it may indeed happen that all agents expect to increase their positions over time in the dynamic case, without contradicting the market clearing condition.
} 
To demonstrate this in more detail, we prohibit short-selling $\left(\alpha_{-}=0\right)$, impose a positive cost for long positions $\left(\alpha_{+}=1\right)$, and construct an example where some agents expect to increase their positions over time but no agent expects to decrease their position. To obtain explicit formulas despite the nonlinear context, we consider the limiting case of zero volatility, and show later (Proposition 4.7) that this is indeed the continuous limit for equilibria with small volatility coefficients $\sigma_{i}$. Similarly as in Remark 4.5, the zero volatility case violates our standing assumption that the coefficients are uniformly parabolic (and indeed $v$ is not smooth in our example), but the formulas we present can be verified by direct calculation.

Example 4.6. Consider $n=2$ types with volatility coefficients $\sigma_{i}=0$ and constant, opposing drifts

$$
b_{1}=1, \quad b_{2}=-1 .
$$

The payoff function is $f(y)=y^{2}$ and the supply $s>0$ is constant. Moreover, $\alpha_{-}=0$ and $\alpha_{+}=1$. Then, the dynamic equilibrium price is

$$
p_{\text {dyn }}= \begin{cases}x^{2}-s T / 2, & |x|+T / 2 \leq s / 4 \\ (|x|+T)^{2}-s T, & |x|+T / 2>s / 4\end{cases}
$$

and corresponding optimal portfolios in feedback form are given by

$$
\begin{gathered}
\phi_{1}(t, x)= \begin{cases}0, & |x|+(T-t) / 2>s / 4, x<0, \\
s / 2+2 x, & |x|+(T-t) / 2 \leq s / 4, \\
s, & |x|+(T-t) / 2>s / 4, x>0,\end{cases} \\
\phi_{2}(t, x)= \begin{cases}s, & |x|+(T-t) / 2>s / 4, x<0, \\
s / 2-2 x, & |x|+(T-t) / 2 \leq s / 4, \\
0, & |x|+(T-t) / 2>s / 4, x>0 .\end{cases}
\end{gathered}
$$

The static equilibrium price is

$$
p_{\text {sta }}= \begin{cases}x^{2}+T^{2}-s T / 2, & |x| \leq s / 4, \\ x^{2}+T^{2}+2|x| T-s T, & |x|>s / 4,\end{cases}
$$

and corresponding optimal portfolios are given by

$$
q_{1}=\left\{\begin{array}{ll}
0, & x<-s / 4, \\
s / 2+2 x, & |x| \leq s / 4, \\
s, & x>s / 4,
\end{array} \quad q_{2}= \begin{cases}s, & x<-s / 4 \\
s / 2-2 x, & |x| \leq s / 4 \\
0, & x>s / 4\end{cases}\right.
$$


The static equilibrium price exceeds the dynamic price; more precisely,

$$
p_{\text {sta }}-p_{\text {dyn }}= \begin{cases}T^{2}, & |x| \leq s / 4-T / 2, \\ (s / 2-2|x|) T, & s / 4-T / 2<|x|<s / 4, \\ 0, & |x| \geq s / 4 .\end{cases}
$$

The calculations for our examples are carried out in Appendix A, together with the rest of the proofs. Next, we discuss in more detail how the delay effect explains the difference $p_{\text {sta }}-p_{\text {dyn }}$ in this example. To that end, it will be useful to record the portfolios as expected by the agents: since $X(t)=x+b_{i} t$ $Q_{i}$-a.s. and $\Phi_{i}(t)=\phi_{i}(t, X(t))$, we have

$$
\begin{aligned}
& Q_{1} \text {-a.s., } \quad \Phi_{1}(t)= \begin{cases}0, & |x+t|+(T-t) / 2>s / 4, x+t<0, \\
s / 2+2 t+2 x, & |x+t|+(T-t) / 2 \leq s / 4, \\
s, & |x+t|+(T-t) / 2>s / 4, x+t>0,\end{cases} \\
& Q_{2} \text {-a.s., } \quad \Phi_{2}(t)= \begin{cases}s, & |x-t|+(T-t) / 2>s / 4, x-t<0, \\
s / 2+2 t-2 x, & |x-t|+(T-t) / 2 \leq s / 4, \\
0, & |x-t|+(T-t) / 2>s / 4, x-t>0 .\end{cases}
\end{aligned}
$$

Below, we abuse this notation and simply write $\Phi_{1}(t)$ and $\Phi_{2}(t)$ for the expressions on the right hand side. We consider various intervals for the initial value $x$; by symmetry, we may focus on $x \geq 0$ without loss of generality. We also assume that $s>T$, mainly to avoid distinguishing even more cases.

Case 1: $x \geq s / 4+T$. In this regime, the expected dynamic portfolios $\Phi_{1}$ and $\Phi_{2}$ are constant, and thus the delay option is never exercised. The static portfolios coincide with their initial values, $q_{1}=s=\Phi_{1}(0)$ and $q_{2}=$ $0=\Phi_{2}(0)$, and the static and dynamic prices are equal: $p_{\text {sta }}=p_{\text {dyn }}$.

Case 2: $s / 4 \leq x<s / 4+T$. As before, $q_{1}=s=\Phi_{1}(0)$ and $\Phi_{1}$ is constant. However, $\Phi_{2}(t)$ equals zero initially but may become positive for $t$ close to $T$ (for suitable parameter values). Nevertheless, type 2 does not choose to anticipate her trading in the static case, because the cost-of-carry outweighs the expected gains - we still have $q_{2}=0=\Phi_{2}(0)$ and $p_{\text {sta }}=p_{\text {dyn }}$.

Case 3: $(s / 4-T / 2)^{+}<x<s / 4$. Once again, $\Phi_{1} \equiv s$ is constant, $\Phi_{2}(0)=0$, and $\Phi_{2}$ increases for some $t>0$. Furthermore, the increase in type 2's position is larger for smaller $x$. Type 2 now does anticipate some of that increase in the static case and for this reason $p_{\text {dyn }}$ is now too low to be an equilibrium price. The increase in price changes the optimal portfolio for agents of type 1 . We are in the mixed case where portfolios and prices adjust. Type 1 decreases his initial position to $q_{1}=s / 2+2 x<s=\Phi_{1}(0)$ and type 2 
increases her position to $q_{2}=s / 2-2 x>0=\Phi_{2}(0)$. At the same time, the static equilibrium price is augmented, $p_{\text {sta }}-p_{\text {dyn }}=(2 x-s / 2) T>0$. As $x$ decreases from $s / 4$ to $s / 4-T / 2$, this difference increases linearly from 0 to $T^{2}$, and the portfolios $\left(q_{1}, q_{2}\right)$ change linearly from $(\mathrm{s}, 0)$ to $(s-T, T)$. In summary, the elimination of the delay option in the static case results in portfolio adjustments and a price increase.

Case 4: $0 \leq x \leq s / 4-T / 2$. In this last regime, both $\Phi_{1}$ and $\Phi_{2}$ are increasing in time, so both types are exercising the delay option when re-trading is allowed and have an anticipatory motive when they can only trade at $t=0$. The initial dynamic portfolios are $\Phi_{1}(0)=s / 2+2 x>0$ and $\Phi_{2}(0)=s / 2-2 x>0$. Since both types want to anticipate in the static case, the static price must be higher. More precisely, the aggregate excess demand at price $p_{\text {dyn }}$ equals $2 T^{2}$ and thus is independent of $x$. Since we are in the region where both types have positive demand, the marginal effect of an increase in price is -1 , for each type. Thus, the price adjustment that is necessary to clear the static market is exactly $T^{2}$ for every value of $x$ in this region.

Finally, we show that the conclusions of the example also hold when volatilities are small but positive, rather than vanishing.

Proposition 4.7. Consider the setting of Example 4.6 with constant volatilities $\sigma:=\sigma_{1}=\sigma_{2} \geq 0$ and denote the corresponding static and dynamic equilibrium prices by $p_{\mathrm{sta}}^{\sigma}$ and $p_{\mathrm{dyn}}^{\sigma}$, respectively. Then, $p_{\mathrm{sta}}^{\sigma} \downarrow p_{\mathrm{sta}}^{0}$ and $p_{\mathrm{dyn}}^{\sigma} \downarrow p_{\mathrm{dyn}}^{0}$ as $\sigma \downarrow 0$. As a consequence, we have

$$
p_{\text {sta }}^{\sigma}-p_{\text {dyn }}^{\sigma} \rightarrow \begin{cases}T^{2}, & |x| \leq s / 4-T / 2 \\ (s / 2-2|x|) T, & s / 4-T / 2<|x|<s / 4 \\ 0, & |x| \geq s / 4\end{cases}
$$

The above example of the delay effect should be contrasted with Proposition 4.3, where we have seen that when there is no cost-of-carry for long positions $\left(\alpha_{+}=\infty\right)$, the dynamic equilibrium price always exceeds the static one, even if short-selling is possible. We have argued that this is due to the most optimistic agent holding the entire market. The following example illustrates the mechanics of the delay option in this situation.

Example 4.8. Let $\alpha_{+}=\infty$ and $\alpha_{-}=1$. We consider $n=2$ types with drift coefficients

$$
b_{1}=1, \quad b_{2}=0
$$

and volatility coefficients $\sigma_{1}=\sigma_{2}=0$. The payoff is $f(y)=y^{2}$ and the initial value is $x=0$, so that the first type is more optimistic at any time. 
As in Proposition 4.2, the static equilibrium price given by the optimist's expectation $e_{1}=E_{1}[f(X(T))]=T^{2}$. Following Proposition 3.4, the same holds for the dynamic price, so that $p_{\text {sta }}=p_{\text {dyn }}$. The static and dynamic portfolios of the pessimist are given by

$$
q_{2}=T^{-1}\left(e_{2}-p_{\text {sta }}\right)=-T, \quad \phi_{2}(t, x)=\partial_{t} v(t, x)=-2(x+T-t) .
$$

Under $Q_{2}$, the state process $X \equiv 0$ is constant, so that $\Phi_{2}(t)=\phi_{2}(t, X(t))=$ $-2(T-t)$ a.s. Thus, the static position $q_{2}=-T$ anticipates some of the increase from $\Phi_{2}(0)=-2 T$ to $\Phi_{2}(T)=0$. However, this does not affect the static equilibrium price because an optimistic agent is indifferent to the size of her (nonnegative) position - the absence of a cost-of-carry for long positions allows the portfolios to adjust without affecting the prices.

\subsection{Illustrating the Effect of Shorting}

The following example illustrates that when shorting is allowed, the static price may exceed the dynamic price - this is quite natural once we observe the symmetry between optimists and pessimists in the extreme case $\alpha_{-}=\alpha_{+}$. The difference between the dynamic price and the static price has been identified as the size of the "speculative bubble" in the previous literature. If we maintain this identification, this example can be used to illustrate how lowering the cost of shorting can lead not only to a bubble implosion but even to a negative bubble.

Example 4.9. To facilitate the computations we assume symmetric costsof-carry $\alpha_{-}=\alpha_{+}=1$. Consider $n=2$ types with constant coefficients $b_{i} \in \mathbb{R}$ and $\sigma_{i}>0$, and an asset in zero aggregate supply with payoff $f(y)=y^{2}$. Writing $\Sigma^{2}:=\left(\sigma_{1}^{2}+\sigma_{2}^{2}\right) / 2$ and $\mu:=\left(b_{1}+b_{2}\right) / 2$, the dynamic and static equilibrium prices at $t=0$ for the initial value $X(0)=x$ are

$$
\begin{aligned}
p_{\text {dyn }} & =x^{2}+2 x \mu T+\Sigma^{2} T+\left(\frac{b_{1}+b_{2}}{2}\right)^{2} T^{2}, \\
p_{\text {sta }} & =x^{2}+2 x \mu T+\Sigma^{2} T+\frac{b_{1}^{2}+b_{2}^{2}}{2} T^{2} ;
\end{aligned}
$$

in particular,

$$
p_{\text {dyn }}-p_{\text {sta }}=\left[\left(\frac{b_{1}+b_{2}}{2}\right)^{2}-\frac{b_{1}^{2}+b_{2}^{2}}{2}\right] T^{2} \leq 0 .
$$


The optimal dynamic and static portfolios are given by

$$
\begin{aligned}
\phi_{i}(t, x) & =x\left(b_{i}-b_{j}\right)+\frac{1}{2}(T-t)\left(b_{i}^{2}-b_{j}^{2}\right)+\frac{1}{2}\left(\sigma_{i}^{2}-\sigma_{j}^{2}\right), \\
q_{i} & =x\left(b_{i}-b_{j}\right)+\frac{1}{2} T\left(b_{i}^{2}-b_{j}^{2}\right)+\frac{1}{2}\left(\sigma_{i}^{2}-\sigma_{j}^{2}\right),
\end{aligned}
$$

where $j=2$ if $i=1$ and vice versa; in particular, the demands at $t=0$ coincide. In the special case $b_{1}=b_{2}$ where all agents agree on the drift, we have $p_{\text {dyn }}=p_{\text {sta }}$ and the demands coincide at all times. Whenever $b_{1} \neq b_{2}$, a continuity result similar to the results established in Section 3.2 guarantees that $p_{\text {dyn }}<p_{\text {sta }}$ for cost parameters close to $\alpha_{-}=\alpha_{+}=1$.

To obtain some intuition for this example, consider the case were $\sigma_{1}=\sigma_{2}$, $b_{1}>0$ and $b_{2}=0$. Then if $x>0$, type 1 is long and type 2 is short when re-trading is allowed. Notice that an agent who is short expects on average to cover some of her shorts in the future. When re-trading is ruled out, she prefers to cut her short position at time zero. This would place upward pressure on the static price. The long party also expects to reduce his position if $x$ would stay constant, but because $b_{1}>0$, he expects the state $X(t)$ to grow, thus dampening his need to anticipate the reduction when re-trading is ruled out. In other words, the long party values the resale option less than the short party values the repurchase option. As a result, the static market presents excess demand at price $p_{\text {dyn }}$ and thus the static price must rise to clear the market.

\section{Conclusion}

In this paper, we considered a continuous-time model of trading among riskneutral agents with heterogeneous beliefs. Agents face quadratic costs-ofcarry and as a consequence, their marginal valuation of the asset decreases when the magnitude of their position increases, as it would be the case for risk-averse agents. In previous models of heterogeneous beliefs, it was assumed that agents face a constant marginal cost-of-carry for a positive position and an infinite cost for a negative position. As a result, buyers benefit from a resale option and are willing to pay for an asset in excess of their own valuation of the dividends of that asset. Moreover, the supply does not affect the equilibrium price. We show that when buyers face an increasing marginal cost-of-carry, in equilibrium, they may also value an option to delay. We illustrate with an example that even when shorting is impossible, this delay option may cause the market to equilibrate below the price that would prevail if agents were restricted to buy-and-hold strategies. 
Moreover, we introduce the possibility of short-selling and show how this gives pessimists the analogous options. In our model, the price depends on the supply. We characterize the unique equilibrium of our model as the solution to a Hamilton-Jacobi-Bellman of a novel form and use this to derive several comparative statics: the price decreases with an increase in the supply of the asset, with an increase in the cost of carrying long positions, and with a decrease in the cost of carrying short positions. The conclusions of earlier models are shown to hold in a limiting case of the model when the cost-of-carry for long positions tends to zero.

In our model the demand for the asset is satisfied by the sum of two components - the exogenous supply and the short positions of the market participants. While the shorts are determined endogenously, the supply is independent of the current price and the beliefs of agents. It would be interesting to model this supply as originating from parties solving an appropriate optimization problem.

\section{A Proofs}

This appendix collects the proofs for the results in Sections 2-4.

\section{A.1 Proofs for Section 2}

Before proving the main result of Theorem 2.1, we record two lemmas for later reference. The first one guarantees the passage from almost-sure to pointwise identities.

Lemma A.1. For all $i \in\{1, \ldots, n\}$ and all $t \in(0, T]$, the support of $X(t)$ under $Q_{i}$ is the full space $\mathbb{R}^{d}$.

Proof. Since $b_{i}$ is bounded and $\sigma_{i}^{2}$ is uniformly parabolic, the support of $Q_{i}$ in $\Omega$ is the set of all paths $\omega \in C\left([0, T], \mathbb{R}^{d}\right)$ with $\omega(0)=x$; see (Stroock and Varadhan, 1972, Theorem 3.1). The claim is a direct consequence.

The second lemma provides an expression for the optimal portfolios.

Lemma A.2. Let $v \in C_{b}^{1,2}$ and consider the (price) process $P(t)=v(t, X(t)$ ). The portfolio defined by $\Phi_{i}(t)=\phi_{i}(t, X(t))$, where

$$
\phi_{i}(t, x)=\alpha_{\operatorname{sign}\left(\mathcal{L}^{i} v(t, x)\right)} \mathcal{L}^{i} v(t, x),
$$

is the unique $e^{21}$ optimal portfolio for type $i$.

\footnotetext{
${ }^{21}$ We recall that uniqueness is understood up to $\left(Q_{i} \times d t\right)$-nullsets.
} 
Proof. We note that $\Phi_{i}$ is admissible since $v \in C_{b}^{1,2}$. Let $\Phi$ be any admissible portfolio. By Itô's formula,

$\int_{0}^{T} \Phi(t) d P(t)-\int_{0}^{T} c(\Phi(t)) d t=\int_{0}^{T}\left\{\Phi(t) \mathcal{L}^{i} v(t, X(t))-c(\Phi(t))\right\} d t+M_{i}(T)$

where $M_{i}(T)$ is the terminal value of a (true) martingale with vanishing expectation; recall that $\sigma_{i}$ and $\partial_{x} v$ are bounded. Thus, the expected final payoff (2.3) is given by

$$
E_{i}\left[\int_{0}^{T}\left\{\Phi(t) \mathcal{L}^{i} v(t, X(t))-c(\Phi(t))\right\} d t\right] .
$$

As a result, $\Phi$ is optimal if and only if it maximizes the above integrand (up to $\left(Q_{i} \times d t\right)$-nullsets). The unique maximizer is given by $\Phi_{i}$, and the claim follows.

We can now prove the main result on the pricing PDE.

Proof of Theorem 2.1. (a) We first show that a given equilibrium price function $v \in C_{b}^{1,2}$ solves the PDE. Since $v(T, X(T))=f(X(T)) Q_{i}$-a.s. for all $i$, the terminal condition $v(T, \cdot)=f$ follows from Lemma A.1. At any state $(t, x)$, we introduce the set

$$
I_{*}(t, x)=\left\{i \in\{1, \ldots, n\}: \mathcal{L}^{i} v(t, x)<0\right\} .
$$

Next, we recall the unique optimal portfolios $\Phi_{i}$ from Lemma A.2. Using again Lemma A.1, the market clearing condition $\sum_{i} \Phi_{i}=S$ can be stated as

$$
\alpha_{-} \sum_{i \in I_{*}} \mathcal{L}^{i} v+\alpha_{+} \sum_{i \in I_{*}^{c}} \mathcal{L}^{i} v=s
$$

If $i \in I_{*}$, then $\mathcal{L}^{i} v \leq 0$ and $\alpha_{-} \leq \alpha_{+}$implies $\alpha_{-} \mathcal{L}^{i} v \geq \alpha_{+} \mathcal{L}^{i} v$. Conversely, if $i \in I_{*}^{c}$, then $\mathcal{L}^{i} v \geq 0$ and $\alpha_{+} \mathcal{L}^{i} v \geq \alpha_{-} \mathcal{L}^{i} v$. It follows that the set $I_{*}$ of (A.2) maximizes the left hand side of (A.3) among all subsets $I \subseteq\{1, \ldots, n\}$. That is,

$$
\max _{I \subseteq\{1, \ldots, n\}}\left(\alpha_{-} \sum_{i \in I} \mathcal{L}^{i} v+\alpha_{+} \sum_{i \in I^{c}} \mathcal{L}^{i} v-s\right)=0
$$

and the set $I_{*}$ is a maximizer, or equivalently,

$$
\max _{I \subseteq\{1, \ldots, n\}} \frac{1}{|I| \alpha_{-}+\left|I^{c}\right| \alpha_{+}}\left(\alpha_{-} \sum_{i \in I} \mathcal{L}^{i} v+\alpha_{+} \sum_{i \in I^{c}} \mathcal{L}^{i} v-s\right)=0 .
$$


After plugging in the definition of $\mathcal{L}^{i} v$ and using the definitions of $\mu_{I}, \Sigma_{I}$ and $\kappa_{I}$ in (2.6)-(2.8), this is the desired PDE (2.5).

(b) Conversely, let $v \in C_{b}^{1,2}$ be a solution of the PDE (2.5) with terminal condition $f$ and define $\Phi_{i}, \phi_{i}$ as in part (i) of Theorem 2.1. Then, the terminal condition $v(T, X(T))=f(X(T))$ is satisfied and $\Phi_{i}$ are optimal by Lemma A.2. Since $v$ is a solution of the equivalent PDE (A.4) and $I_{*}$ of (A.2) is a maximizer, we have that

$$
\sum_{1 \leq i \leq n} \phi_{i}=\alpha_{-} \sum_{i \in I_{*}} \mathcal{L}^{i} v+\alpha_{+} \sum_{i \in I_{*}^{c}} \mathcal{L}^{i} v=s
$$

that is, the market clears. This shows that $v$ is an equilibrium price function.

(c) Since (a) and (b) established a one-to-one correspondence between equilibria and solutions of the $\operatorname{PDE}(2.5)$ with terminal condition $f$, it remains to observe that the latter has a unique solution in $C_{b}^{1,2}$. Indeed, existence holds by (Krylov, 1987, Theorem 6.4.3, p. 301); the conditions in the cited theorem can be verified as in (Krylov, 1987, Example 6.1.4, p. 279). Uniqueness holds by the comparison principle for parabolic PDEs; see (Fleming and Soner, 2006, Theorem V.9.1, p.223).

Next, we prove the optimal control representation for the equilibrium price function.

Proof of Proposition 2.3. By Theorem 2.1, the function $v \in C_{b}^{1,2}$ is a solution of the PDE (2.5) which is the Hamilton-Jacobi-Bellman equation of the control problem (2.10). Moreover, $I_{*}(t, x)$ maximizes the Hamiltonian as noted after (A.4). Thus, the verification theorem of stochastic control, cf. (Fleming and Soner, 2006, Theorem IV.3.1, p. 157), shows that $v$ is the value function and $\mathcal{I}_{*}$ is an optimal control.

It remains to analyze the planner's problem.

Proof of Theorem 2.4. (i) In what follows, we fix the assignment $\mathcal{I}(t)=$ $I(t, X(t))$ and often omit the dependence on $\mathcal{I}$ in the notation. Suppose that $w$ is an equilibrium price function; we show that $w$ satisfies (2.12). As in Lemma A.2, the unique optimal portfolio for agent $i$ is given by $\Phi_{i}(t)=\phi_{i}(t)$, where

$$
\phi_{i}(t, x)=\alpha_{i}(t, x) \mathcal{L}^{i} w(t, x) .
$$

The market clearing condition then implies

$$
\alpha_{-} \sum_{i \in I} \mathcal{L}^{i} w+\alpha_{+} \sum_{i \in I^{c}} \mathcal{L}^{i} w=s
$$


which is equivalent to

$$
\frac{1}{|I| \alpha_{-}+\left|I^{c}\right| \alpha_{+}}\left(\alpha_{-} \sum_{i \in I} \mathcal{L}^{i} w+\alpha_{+} \sum_{i \in I^{c}} \mathcal{L}^{i} w-s\right)=0
$$

or

$$
\partial_{t} w+\mu_{I} \partial_{x} w+\frac{1}{2} \operatorname{Tr} \Sigma_{I}^{2} \partial_{x x} w-\kappa_{I}=0 .
$$

Given the terminal condition $f$, this linear PDE has a unique solution in $C_{b}^{1,2}$ which, by the Feynman-Kac formula (Karatzas and Shreve, 1991, Theorem 5.7.6, p. 366), has the representation (2.12).

Conversely, starting with the unique solution $w=v_{\mathcal{I}} \in C_{b}^{1,2}$ of that PDE, reversing the above arguments shows that $w$ is an equilibrium price function, and that completes the proof of (i).

(ii) In view of $(2.12),(2.10)$ and Proposition 2.3, the supremum of $v_{\mathcal{I}}(t, x)$ over all assignments $\mathcal{I}(t)=I(t, X(t))$ is given by $V(t, x)$ and $(2.11)$ is an optimal assignment in feedback form.

\section{A.2 Proofs for Section 3}

We start with the comparative statics for the dependence of the price on the supply.

Proof of Proposition 3.1. Since the function $s$ enters linearly in the running cost (2.8) of the control problem (2.10) and nowhere else, it follows immediately that the value function $V$ is monotone decreasing in $s$, and now the claim follows from Proposition 2.3.

Next, we consider the dependence on the cost coefficients.

Proof of Proposition 3.2 and Remark 3.3. Let $\alpha_{-} \leq \alpha_{+}$and $\alpha_{-}^{\prime} \leq \alpha_{+}^{\prime}$ be two pairs of cost coefficients and let $v$ and $v^{\prime}$ be the corresponding equilibrium price functions. Let $I_{*}$ be the optimal feedback control for $\alpha_{ \pm}$as defined in (A.2), then by (A.4) we have

$$
\alpha_{-} \sum_{i \in I_{*}} \mathcal{L}^{i} v+\alpha_{+} \sum_{i \in I_{*}^{c}} \mathcal{L}^{i} v-s=0
$$

If $\alpha_{-}^{\prime} \leq \alpha_{-}$and $\alpha_{+}^{\prime} \geq \alpha_{+}$, then $\sum_{i \in I_{*}} \mathcal{L}^{i} v \leq 0$ and $\sum_{i \in I_{*}^{c}} \mathcal{L}^{i} v \geq 0$ yield that

$$
\alpha_{-}^{\prime} \sum_{i \in I_{*}} \mathcal{L}^{i} v+\alpha_{+}^{\prime} \sum_{i \in I_{*}^{c}} \mathcal{L}^{i} v-s \geq 0
$$


In the special case where $s \equiv 0$, this conclusion also holds under the weaker condition that $\alpha_{+} / \alpha_{-} \leq \alpha_{+}^{\prime} / \alpha_{-}^{\prime}$, which covers the case (iii), and the same holds under the conditions of Remark 3.3. It then follows that

$$
\max _{I \subseteq\{1, \ldots, n\}}\left(\alpha_{-}^{\prime} \sum_{i \in I} \mathcal{L}^{i} v+\alpha_{+}^{\prime} \sum_{i \in I^{c}} \mathcal{L}^{i} v-s\right) \geq 0
$$

which is a version of (A.4) with inequality instead of equality, for the coefficients $\alpha_{ \pm}^{\prime}$. Following the same steps as after (A.4), we deduce that

$$
\partial_{t} v+\sup _{I \subseteq\{1, \ldots, n\}}\left(\mu_{I}^{\prime} \partial_{x} v+\frac{1}{2} \operatorname{Tr} \Sigma_{I}^{\prime 2} \partial_{x x} v-\kappa_{I}^{\prime}\right) \geq 0
$$

where $\mu_{I}^{\prime}, \Sigma_{I}^{\prime}, \kappa_{I}^{\prime}$ are defined as in (2.6)-(2.8) but with $\alpha_{ \pm}^{\prime}$ instead of $\alpha_{ \pm}$. In other words, $v$ is a subsolution ${ }^{22}$ of the PDE satisfied by $v^{\prime}$. As $v$ and $v^{\prime}$ satisfy the same terminal condition $f$, the comparison principle (Fleming and Soner, 2006, Theorem V.9.1, p. 223) implies that $v \leq v^{\prime}$.

We continue with our result on the limit $\alpha_{+} \rightarrow \infty$.

Proof of Proposition 3.4. We first notice that since $s \geq 0$, the optimal set $I_{*}$ of (A.2) for the Hamiltonian of the PDE (2.5) must satisfy $\left|I_{*}\right|<n$ due to the market clearing condition - at least one agent has to hold a nonnegative position. As a result, the PDE (2.5) remains the same if the supremum is taken only over sets $I$ with $\left|I^{c}\right|>0$.

Taking that into account, the limiting PDE for (2.5) as $\alpha_{+} \rightarrow \infty$ is

$$
\partial_{t} v+\sup _{\emptyset \neq J \subseteq\{1, \ldots, n\}} \frac{1}{|J|} \sum_{i \in J}\left(b_{i} \partial_{x} v+\frac{1}{2} \operatorname{Tr} \sigma_{i}^{2} \partial_{x x} v\right)=0 .
$$

Notice that given a set of real numbers, the largest average over a subset is in fact equal to the largest number in the set. As a result, (A.7) coincides with (3.1). Using again (Krylov, 1987, Theorem 6.4.3, p. 301) and (Fleming and Soner, 2006, Theorem V.9.1, p. 223), this equation has a unique solution $v^{\infty} \in C_{b}^{1,2}$ for the terminal condition $f$, and the solution is independent of $\alpha_{-}$and $s$ since these quantities do not appear in (3.1).

To see that $v^{\alpha_{-}, \alpha_{+}}(t, x) \rightarrow v^{\infty}(t, x)$, one can apply a PDE technique called the Barles-Perthame procedure to the equations under consideration; see (Fleming and Soner, 2006, Section VII.3). Alternately, and to give a

\footnotetext{
${ }^{22}$ Note that the sign convention chosen here is opposite to the one of Fleming and Soner (2006), so that a subsolution corresponds to the inequality $\geq 0$ in the PDE.
} 
more concise proof, we may use the representation $(2.10)$ of $v^{\alpha_{-}, \alpha_{+}}$as a value function as well as the corresponding representation for $v^{\infty}$. Then, a result on the stability of value functions, cf. (Krylov, 1980, Corollary 3.1.13, p. 138), shows that $v^{\alpha_{-}, \alpha_{+}} \rightarrow v^{\infty}$ locally uniformly; that is,

$$
\sup _{(t, x) \in[0, T] \times K}\left|v^{\alpha_{-}, \alpha_{+}}(t, x)-v^{\infty}(t, x)\right| \rightarrow 0
$$

for any compact set $K \subseteq \mathbb{R}^{d}$. The monotonicity property of the limit follows from Proposition 3.2.

Finally, we turn to the limit $\alpha_{-} \rightarrow 0$.

Proof of Proposition 3.6. The arguments are similar to the ones for Proposition 3.4. In this case, the limiting PDE for (2.5) as $\alpha_{-} \rightarrow 0$ is (3.3). As in the proof of Proposition 3.4 we have that the limiting PDE has a unique solution $v^{0, \alpha_{+}} \in C_{b}^{1,2}$ and $v^{\alpha_{-}, \alpha_{+}}(t, x) \rightarrow v^{0, \alpha_{+}}(t, x)$ locally uniformly, with monotonicity in $\alpha_{-}$. In the special case where $s=0$, the PDE (3.3) coincides with (A.7), and thus with (3.1) as shown in the proof of Proposition 3.4.

\section{A.3 Proofs for Section 4}

We first prove our formula for the static equilibrium price.

Proof of Proposition 4.1. We set $e_{i}=E_{i}[f(X(T))]$. Given any price $p$, the expected net payoff for agent $i$ using portfolio $q$ is

$$
q\left(e_{i}-p\right)-\frac{T}{2 \alpha_{\operatorname{sign}(q)}} q^{2}
$$

and the unique maximizer is $q_{i}=\alpha_{\operatorname{sign}\left(e_{i}-p\right)} T^{-1}\left(e_{i}-p\right)$ as stated in (4.2).

Let $p$ be a static equilibrium price. Setting $I_{*}=\left\{i \in\{1, \ldots, n\}: e_{i}<p\right\}$, the market clearing condition $\sum_{i} q_{i}=s$ for these optimal portfolios yields

$$
\alpha_{-} \sum_{i \in I_{*}}\left(e_{i}-p\right)+\alpha_{+} \sum_{i \in I_{*}^{c}}\left(e_{i}-p\right)=s T
$$

and we observe that $I_{*}$ maximizes the left hand side; that is,

$$
\max _{I \subseteq\{1, \ldots, n\}}\left(\alpha_{-} \sum_{i \in I}\left(e_{i}-p\right)+\alpha_{+} \sum_{i \in I^{c}}\left(e_{i}-p\right)-s T\right)=0 .
$$

This is equivalent to the claimed representation (4.1) for $p$. 
Conversely, define $p$ by (4.1) and $q_{i}$ by (4.2), then $q_{i}$ is optimal for agent $i$ as mentioned in the beginning of the proof. Moreover, reversing the above, $p$ satisfies (A.8) and thus

$$
\sum_{i=1}^{n} q_{i}=\alpha_{-} \sum_{i \in I_{*}} T^{-1}\left(e_{i}-p\right)+\alpha_{+} \sum_{i \in I_{*}^{c}} T^{-1}\left(e_{i}-p\right)=s,
$$

establishing market clearing.

We can now deduce the formulas for the limiting cases of the static equilibrium.

Proof of Proposition 4.2. Formula (4.3) follows by taking the limit $\alpha_{+} \rightarrow \infty$ in (4.1). Similarly, (4.4) is obtained by taking the limit $\alpha_{-} \rightarrow 0$ in (4.1).

Next, we show that in the limit $\alpha_{+} \rightarrow \infty$ with no cost on long positions, the dynamic price exceeds the static one.

Proof of Proposition 4.3. By the formula (4.3) for $p_{\mathrm{sta}}^{\infty}$, it suffices to verify that $E_{i}[f(X(T))] \leq p_{\text {dyn }}^{\infty}$ for fixed $i \in\{1, \ldots, n\}$. Indeed, let $u=u_{i} \in C_{b}^{1,2}$ be the unique solution of

$$
\partial_{t} u+b_{i} \partial_{x} u+\frac{1}{2} \operatorname{Tr} \sigma_{i}^{2} \partial_{x x} u=0, \quad u(T, \cdot)=f .
$$

Then, by the Feynman-Kac formula (Karatzas and Shreve, 1991, Theorem 5.7.6, p. 366), we have $u(0, x)=E_{i}[f(X(T))]$. Moreover, $u$ is clearly a subsolution of the $\operatorname{PDE}$ (3.1) for $v^{\infty}$, and now the comparison principle (Fleming and Soner, 2006, Theorem V.9.1, p. 223) yields that $E_{i}[f(X(T))]=$ $u(0, x) \leq v^{\infty}(0, x)=p_{\text {dyn }}^{\infty}$ as claimed.

In what follows, we show that in the limit $\alpha_{-} \rightarrow 0$ where short-selling is prohibited, the same inequality holds, provided one agents holds the static market.

Proof of Proposition 4.4. In view of (4.4), we have $p_{\text {sta }}^{0, \alpha_{+}}=E_{i}[f(X(T))]-\frac{s T}{\alpha_{+}}$ since the maximizing set is $J=\{i\}$. Using again the Feynman-Kac formula (Karatzas and Shreve, 1991, Theorem 5.7.6, p. 366), we deduce that $p_{\text {sta }}^{0, \alpha_{+}}=$ $u(0, x)$ where $u \in C_{b}^{1,2}$ is the solution of

$$
\partial_{t} u+b_{i} \partial_{x} u+\frac{1}{2} \operatorname{Tr} \sigma_{i}^{2} \partial_{x x} u-\frac{s T}{\alpha_{+}}=0, \quad u(T, \cdot)=f .
$$

In particular, $u$ is a subsolution of the $\operatorname{PDE}(3.3)$ for $v^{0, \alpha_{+}}$, and now the comparison principle (Fleming and Soner, 2006, Theorem V.9.1, p. 223) yields that $p_{\text {sta }}^{0, \alpha_{+}}=u(0, x) \leq v^{0, \alpha_{+}}(0, x)=p_{\text {dyn }}^{0, \alpha_{+}}$as desired. 
We turn to our example where the static price exceeds the dynamic one due to the delay effect.

Proofs for Example 4.6. We begin with the static case. For later use, we consider the more general situation where $\sigma:=\sigma_{1}=\sigma_{2}$ may be positive (but constant). We have $e_{i}=E_{i}[f(X(T))]=x^{2}+2 x b_{i} T+T^{2}+\sigma^{2} T$ and thus, as in (4.4), the static price $p_{\text {sta }}$ is

$$
\begin{aligned}
p_{\text {sta }} & =\max _{\emptyset \neq J \subseteq\{1,2\}}\left(\frac{1}{|J|} \sum_{i \in J} e_{i}-\frac{s T}{|J|}\right) \\
& =x^{2}+\sigma^{2} T+\max \left\{T^{2}-s T / 2, T^{2}+2|x| T-s T\right\}
\end{aligned}
$$

or

$$
p_{\text {sta }}= \begin{cases}x^{2}+\sigma^{2} T+T^{2}-s T / 2, & |x| \leq s / 4 \\ x^{2}+\sigma^{2} T+T^{2}+2|x| T-s T, & |x|>s / 4\end{cases}
$$

and the portfolios $q_{i}$ are as stated in Example 4.6.

We turn to the dynamic case and restrict to $\sigma=0$. The limiting equation for $(3.3)$ is

$$
\partial_{t} v+\max \left(\left|\partial_{x} v\right|-s,-s / 2\right)=0, \quad v(T, \cdot)=f .
$$

In analogy to Proposition 2.3, this can be seen as the Hamilton-Jacobi equation of a deterministic control problem where the drift $\mu$ of the controlled state $d X(t)=\mu(t, X(t)) d t$ can be chosen to be \pm 1 or 0 and the running cost is $s$ or $s / 2$, respectively. We can check directly that an optimal control for this problem is

$$
\mu(t, x)= \begin{cases}\operatorname{sign}(x), & |x|+(T-t) / 2>s / 4, \\ 0, & |x|+(T-t) / 2 \leq s / 4,\end{cases}
$$

and then the value function is found to be

$$
v(t, x)= \begin{cases}(|x|+T-t)^{2}-s(T-t), & |x|+(T-t) / 2>s / 4, \\ x^{2}-s(T-t) / 2, & |x|+(T-t) / 2 \leq s / 4\end{cases}
$$

Indeed, $v$ is continuous and the unique viscosity ${ }^{23}$ solution of (A.10). The indicated formulas for $p_{\text {dyn }}-p_{\text {sta }}=v(0, x)-p_{\text {sta }}$ and for the optimal controls $\phi_{i}$ follow.

\footnotetext{
${ }^{23}$ As is often the case for deterministic control problems, the value function is not $C^{1,1}$ and (A.10) has no classical solution.
} 
Next, we prove the continuity of the prices in the small volatility limit.

Proof of Proposition 4.7. For the static case, the formula for $p_{\text {sta }}^{\sigma}$ stated in (A.9) shows that $p_{\text {sta }}^{\sigma}-p_{\text {sta }}^{0}=\sigma^{2} T \downarrow 0$. Turning to the dynamic case, we first show that $p_{\text {dyn }}^{\sigma}=v^{\sigma}(0, x)$ is monotone with respect to $\sigma$. Since $f$ is convex, $x \mapsto v^{\sigma}(t, x)$ is convex and thus $\partial_{x x} v^{\sigma} \geq 0$. Given $\sigma_{1} \geq \sigma_{2}>0$, it follows that $v^{\sigma_{2}}$ is a subsolution to the equation (3.3) for $v^{\sigma_{1}}$, and thus the comparison principle for parabolic PDEs implies that $v^{\sigma_{1}} \geq v^{\sigma_{2}}$. To see that $v^{\sigma}(t, x) \rightarrow v^{0}(t, x)$ and in particular $p_{\mathrm{dyn}}^{\sigma} \rightarrow p_{\mathrm{dyn}}^{0}$, we may again use a general result on the stability of value functions; cf. (Krylov, 1980, Corollary 3.1.13, p. 138).

It remains to provide the calculations for our symmetric example with $\alpha_{-}=\alpha_{+}=1$.

Proofs for Example 4.9. Following Remark 3.5, the equilibrium price function in the dynamic case is

$$
v(t, x)=E\left[f\left(x+\mu \tau+\Sigma B_{\tau}\right)\right], \quad \text { where } \quad \tau:=T-t
$$

and $B_{\tau}$ is a centered Gaussian with variance $\tau$. As $f(y)=y^{2}$,

$$
v(t, x)=x^{2}+2 x \mu \tau+\mu^{2} \tau^{2}+\Sigma^{2} \tau
$$

and the optimal portfolios in feedback form are given by

$$
\phi_{i}(t, x)=\mathcal{L}^{i} v(t, x)=x\left(b_{i}-b_{j}\right)+\frac{1}{2} \tau\left(b_{i}^{2}-b_{j}^{2}\right)+\frac{1}{2}\left(\sigma_{i}^{2}-\sigma_{j}^{2}\right) .
$$

For the static case, we have

$$
e_{i}=x^{2}+2 x b_{i} T+b_{i}^{2} T^{2}+\sigma_{i}^{2} T
$$

and thus

$$
p_{\text {sta }}=\frac{e_{1}+e_{2}}{2}=x^{2}+2 x \mu T+\frac{b_{1}^{2}+b_{2}^{2}}{2} T^{2}+\Sigma^{2} T
$$

as well as

$$
q_{i}=T^{-1}\left(e_{i}-p_{\text {sta }}\right)=T^{-1} \frac{e_{i}-e_{j}}{2}=x\left(b_{i}-b_{j}\right)+\frac{1}{2} T\left(b_{i}^{2}-b_{j}^{2}\right)+\frac{1}{2}\left(\sigma_{i}^{2}-\sigma_{j}^{2}\right) .
$$




\section{B Adding Linear Costs}

In this section, we generalize the cost-of-carry by adding linear terms and discuss the corresponding changes in our main results. Broadly speaking, the generalized model does not alter the economic conclusions.

Indeed, let

$$
c(y)= \begin{cases}\frac{1}{2 \alpha_{+}} y^{2}+\beta_{+} y, & y \geq 0, \\ \frac{1}{2 \alpha_{-}} y^{2}+\beta_{-}|y|, & y<0,\end{cases}
$$

where $\beta_{-}, \beta_{+} \geq 0$ are constants; as discussed in the Introduction, the main case of interest is $\beta_{-}>0$ and $\beta_{+}=0$. While this cost function is still strictly convex, it fails to be differentiable at $y=0$ unless $\beta_{-}=\beta_{+}=0$.

Following the proof of Lemma A.2, the optimal portfolio (A.1) then becomes

$$
\phi_{i}(t, x)= \begin{cases}\alpha_{+}\left(\mathcal{L}^{i} v(t, x)-\beta_{+}\right), & \mathcal{L}^{i} v(t, x) \geq \beta_{+}, \\ \alpha_{-}\left(\mathcal{L}^{i} v(t, x)+\beta_{-}\right), & \mathcal{L}^{i} v(t, x) \leq-\beta_{-}, \\ 0, & \text { else. }\end{cases}
$$

That is, there is an interval $\left[-\beta_{-}, \beta_{+}\right]$of values of $\mathcal{L}^{i} v(t, x)$ where it is optimal to have a zero position, due to the kink in the function $c$.

The main PDE (2.5) needs to be adapted correspondingly. Indeed, instead of considering only the group $I$ of agents holding a short position, we now need to distinguish a group $J$ of agents holding a (strict) long positionthe group $J$ may be smaller than the complement $I^{c}$. More precisely, the generalized PDE (2.5) reads as follows (the proof is analogous to Theorem 2.1).

Theorem B.1. The unique equilibrium price function $v \in C_{b}^{1,2}$ can be characterized as the unique solution of the PDE

$\partial_{t} v(t, x)+\sup _{I \cap J=\emptyset}\left(\mu_{I, J}(t, x) \partial_{x} v(t, x)+\frac{1}{2} \operatorname{Tr} \Sigma_{I, J}^{2}(t, x) \partial_{x x} v(t, x)-\kappa_{I, J}(t, x)\right)=0$

on $[0, T) \times \mathbb{R}^{d}$ with terminal condition $v(T, x)=f(x)$, where the supremum is taken over all disjoint subsets $I, J \subseteq\{1, \ldots, n\}$ and the coefficients are defined as

$$
\begin{aligned}
\mu_{I, J}(t, x) & =\frac{\alpha_{-}}{|I| \alpha_{-}+|J| \alpha_{+}} \sum_{i \in I} b_{i}(t, x)+\frac{\alpha_{+}}{|I| \alpha_{-}+|J| \alpha_{+}} \sum_{i \in J} b_{i}(t, x), \\
\Sigma_{I, J}^{2}(t, x) & =\frac{\alpha_{-}}{|I| \alpha_{-}+|J| \alpha_{+}} \sum_{i \in I} \sigma_{i}^{2}(t, x)+\frac{\alpha_{+}}{|I| \alpha_{-}+|J| \alpha_{+}} \sum_{i \in J} \sigma_{i}^{2}(t, x),
\end{aligned}
$$




$$
\kappa_{I, J}(t, x)=\frac{s(t, x)-|I| \alpha_{-} \beta_{-}+|J| \alpha_{+} \beta_{+}}{|I| \alpha_{-}+|J| \alpha_{+}} .
$$

In particular, the additional constants $\beta_{-}, \beta_{+}$enter only through the running cost $\kappa_{I, J}$. It follows that the results on the comparative statics in Propositions 3.1 and 3.2 remain valid; in addition, the equilibrium price function $v$ is increasing with respect to $\beta_{-}$and decreasing with respect to $\beta_{+}$.

In the limiting case of zero cost for long positions, we now need to send $\alpha_{+} \rightarrow \infty$ and $\beta_{+} \rightarrow 0$. Then, the result of Proposition 3.4 is unchanged; i.e., the limiting equilibrium price function is the solution of

$$
\partial_{t} v+\sup _{i \in\{1, \ldots, n\}}\left(b_{i} \partial_{x} v+\frac{1}{2} \operatorname{Tr} \sigma_{i}^{2} \partial_{x x} v\right)=0 .
$$

On the other hand, for the limit $\alpha_{-} \rightarrow 0$ of infinite cost for shorting, the result of Proposition 3.6 changes slightly because the long positions are subject to $\beta_{+}$which becomes an additional running cost in the limiting equation

$$
\partial_{t} v+\sup _{\emptyset \neq J \subseteq\{1, \ldots, n\}}\left(\frac{1}{|J|} \sum_{i \in J} b_{i} \partial_{x} v+\frac{1}{2} \operatorname{Tr} \frac{1}{|J|} \sum_{i \in J} \sigma_{i}^{2} \partial_{x x} v-\frac{s}{|J| \alpha_{+}}-\beta_{+}\right)=0 .
$$

The results for the static equilibrium problem can be generalized with analogous changes.

\section{Heterogeneous Costs}

In this section, we show how the equilibrium of Theorem 2.1 changes if the cost coefficients $\alpha_{-}, \alpha_{+}$depend on the type rather than being the same for all agents. We write $\alpha_{-}^{i}, \alpha_{+}^{i}$ for the coefficients of type $i$. The following result shows that while the structure of the equilibrium remains similar, agents with lower costs have a larger influence on the coefficients of the PDE that determines the equilibrium price.

Theorem C.1. The unique equilibrium price function $v \in C_{b}^{1,2}$ can be characterized as the unique solution of the PDE (2.5) with coefficients

$$
\begin{aligned}
& \mu_{I}(t, x)=\frac{1}{\sum_{i \in I} \alpha_{-}^{i}+\sum_{i \in I^{c}} \alpha_{+}^{i}}\left(\sum_{i \in I} \alpha_{-}^{i} b_{i}(t, x)+\sum_{i \in I^{c}} \alpha_{+}^{i} b_{i}(t, x)\right), \\
& \Sigma_{I}^{2}(t, x)=\frac{1}{\sum_{i \in I} \alpha_{-}^{i}+\sum_{i \in I^{c}} \alpha_{+}^{i}}\left(\sum_{i \in I} \alpha_{-}^{i} \sigma_{i}^{2}(t, x)+\sum_{i \in I^{c}} \alpha_{+}^{i} \sigma_{i}^{2}(t, x)\right),
\end{aligned}
$$




$$
\kappa_{I}(t, x)=\frac{s(t, x)}{\sum_{i \in I} \alpha_{-}^{i}+\sum_{i \in I^{c}} \alpha_{+}^{i}} .
$$

The proof is analogous to Theorem 2.1. As in Lemma A.2, the optimal portfolios are given by $\alpha_{ \pm}^{i} \mathcal{L}^{i} v(t, x)$. Thus, as expected, types with lower costs hold larger positions.

\section{References}

Berestycki, H., C. Bruggeman, R. Monneau, and J. A. Scheinkman. 2016. Bubbles in assets with finite life. Available at SSRN: https://ssrn.com/abstract=2617078 .

Carlos, A., L. Neal, and K. Wandschneider. 2006. Dissecting the anatomy of exchange alley: The dealings of stockjobbers during and after the South Sea bubble. Unpublished paper, University of Illinois .

Cochrane, J. H. 2002. Stocks as money: Convenience yield and the tech-stock bubble. Tech. rep., National Bureau of Economic Research.

Dumas, B., A. Kurshev, and R. Uppal. 2009. Equilibrium portfolio strategies in the presence of sentiment risk and excess volatility. Journal of Finance $64(2): 579-629$.

Fleming, W. H. and H. M. Soner. 2006. Controlled Markov Processes and Viscosity Solutions. Springer, New York, 2nd ed.

Harris, R. 1994. The Bubble Act: Its passage and its effects on business organization. The Journal of Economic History 54 (3):610-627.

Harrison, J. M. and D. M. Kreps. 1978. Speculative investor behavior in a stock market with heterogeneous expectations. Quarterly Journal of Economics 92 (2):323-336.

Hong, H. and J. C. Stein. 2007. Disagreement and the stock market. Journal of Economic Perspectives 21 (2):109-128.

Hong, H., J. A. Scheinkman, and W. Xiong. 2006. Asset float and speculative bubbles. Journal of Finance 61 (3):1073-1117.

Karatzas, I. and S. E. Shreve. 1991. Brownian Motion and Stochastic Calculus. Springer, New York, 2nd ed. 
Kindleberger, C. P. and R. Z. Aliber. 2005. Manias, Panics, and Crashes. John Wiley and Sons, Hoboken.

Krylov, N. V. 1980. Controlled Diffusion Processes. Springer, New York.

. 1987. Nonlinear elliptic and parabolic equations of the second order, vol. 7 of Mathematics and its Applications (Soviet Series). D. Reidel Publishing Co., Dordrecht.

Lewis, M. 2015. The Big Short: Inside the Doomsday Machine. Norton \& Company, New York.

Muhle-Karbe, J. and M. Nutz. 2016. A risk-neutral equilibrium leading to uncertain volatility pricing. Available at SSRN: https://ssrn.com/abstract=2891478.

Ofek, E. and M. Richardson. 2003. Dotcom mania: The rise and fall of internet stock prices. Journal of Finance 58 (3):1113-1138.

Santos, M. S. and M. Woodford. 1997. Rational Asset Pricing Bubbles. Econometrica 65 (1):19-57.

Scheinkman, J. A. 2014. Speculation, Trading, and Bubbles. Columbia University Press, New York.

Scheinkman, J. A. and W. Xiong. 2003. Overconfidence and speculative bubbles. Journal of Political Economy 111:1183-1219.

Stroock, D. W. and S. R. S. Varadhan. 1972. On the support of diffusion processes with applications to the strong maximum principle. In Proceedings of the Sixth Berkeley Symposium on Mathematical Statistics and Probability, Vol. III: Probability theory, 333-359. University of California Press, Berkeley.

Xiong, W. and J. Yu. 2011. The Chinese Warrants Bubble. American Economic Review 101 (6):2723-53. 